\title{
Boundary Mixing and the Dynamics of Three-Dimensional Thermohaline Circulations
}

\author{
JoCHeM MAROTZKe \\ Center for Global Change Science, Department of Earth, Atmospheric, and Planetary Sciences, \\ Massachusetts Institute of Technology, Cambridge, Massachusetts
}

(Manuscript received 6 August 1996, in final form 10 February 1997)

\begin{abstract}
Boundary mixing is implemented in an ocean general circulation model such that the vertical mixing coefficient $k_{v}$ is nonzero only near side boundaries and in convection regions. The model is used in a highly idealized configuration with no wind forcing and very nearly fixed surface density to investigate the three-dimensional dynamics of the thermohaline circulation. For $k_{v}=20 \times 10^{-4} \mathrm{~m}^{2} \mathrm{~s}^{-1}$ and lower, the meridional overturning strength to great accuracy is proportional to $k_{v}^{2 / 3}$; meridional heat transport is proportional to $k_{v}^{1 / 2}$. The circulation patterns resemble those from runs with uniform vertical mixing, but vertical motion is entirely confined to the boundary regions. Near the western boundary, there is upwelling everywhere. Near the eastern boundary, there is a consistent pattern of downwelling above upwelling, with downwelling reaching deeper at high latitudes; this pattern is explained by convection and vertical advective-diffusive balance underneath.

For $k_{v}=30 \times 10^{-4} \mathrm{~m}^{2} \mathrm{~s}^{-1}$ and higher, no steady solutions have been found; the meridional overturning oscillates on a timescale of about 25 years. A time-averaged thermally direct overturning cell is not supported dynamically because convection extends longitudinally across the entire basin, and upwelling near the western boundary does not lead to densities higher than at the eastern boundary.

Assuming uniform upwelling in the west, level isopycnals near the equator, and level isopycnals along the eastern boundary south of the outcropping latitude permits the analytic determination of convection depth at the eastern wall and hence the density difference between the eastern and western walls. This difference is at most one-quarter the surface density difference between high and low latitudes, and agrees in magnitude and latitudinal dependence with the numerical experiments. Scaling arguments estimate overturning strength as of the order of $10 \times 10^{6} \mathrm{~m}^{3} \mathrm{~s}^{-1}$ and confirm the $2 / 3$ power dependence on $k_{v}$. The derivation also gives a dependence of overturning strength with latitude that agrees qualitatively with the numerical results. The scaling for the dependence of meridional heat transport on latitude agrees well with the model results; scaling for heat transport amplitude agrees less well but correctly predicts a weaker dependence on $k_{v}$ than maximum overturning.
\end{abstract}

\section{Introduction}

Munk (1966) already speculated that the horizontally averaged vertical (or diapycnal) diffusivities he estimated from abyssal profiles of temperature and tracers might be due to mixing primarily near horizontal boundaries. This view has been corroborated by the smallness of estimates of diapycnal diffusivities in the thermocline, which are typically smaller by an order of magnitude than Munk's, whether determined by microstructure measurements (e.g., see Gregg 1987, for a review) or from direct tracer dispersion studies (e.g., Ledwell et al. 1993). Hence, it seems necessary to make up for the lack of vigor in thermocline mixing by enhanced mixing near the ocean margins, so that the integral balances hold. Examples of enhanced boundary mixing in

Corresponding author address: Dr. Jochem Marotzke, Dept. of Earth, Atmospheric and Planetary Sciences, Massachusetts Institute of Technology, Room 54-1514, 77 Mass. Ave., Cambridge, MA 02139-4307.

E-mail: jochem@sound.mit.edu isolated basins were presented by Ledwell and Hickey (1995) and Ledwell and Bratkovich (1995); the latter authors report diapycnal mixing near the boundary of the Santa Cruz Basin of $10^{-4} \mathrm{~m}^{2} \mathrm{~s}^{-1}, 10$ times stronger than in the interior.

Where diapycnal mixing occurs, and how strongly, is of paramount importance for the whole field of ocean climate modeling. Bryan (1987) demonstrated that the zonally integrated meridional overturning circulation, a crucial agent of heat transport in the ocean, is very sensitive to the assumed value of vertical diffusivity in an ocean general circulation model (GCM; notice that diapycnal mixing has a horizontal component as well when isopycnals slope). A number of studies addressed the sensitivity of ocean models to stability-dependent diapycnal mixing (Cummins et al. 1990), double-diffusive effects on diapycnal mixing (Gargett and Holloway 1992; Zhang et al. 1997), and the reduction of presumed spurious diapycnal mixing due to incorrect representation of quasi-horizontal eddy stirring (Böning et al. 1995). Surprisingly, however, one very basic question does not seem to have been addressed at all: What are the consequences if the diapycnal mixing in an ocean 
model is assumed zero except near the lateral boundaries? This lack of attention is all the more surprising since Munk inspired a theoretical study of the effects of boundary mixing (Schiff 1966), which appeared simultaneously with the "Abyssal Recipes" (Wunsch 1970, later showed that Schiff's solution contained a mathematical inconsistency that rendered it invalid; this could explain partially why the topic was not followed up). Gregg (1987, p. 5283) posed the question whether a diapycnal diffusivity of less than $10^{-5} \mathrm{~m}^{2} \mathrm{~s}^{-1}$ was small enough so that diapycnal mixing could be ignored in GCMs altogether; he did not, however, explicitly discuss the potentially special role of side boundaries.

This paper investigates first whether "sensible" solutions can be obtained with a GCM that assumes vertical mixing only at the margin (and, through convection, in the mixed layer). The working hypothesis is employed that diapycnal mixing can be approximated by vertical mixing. Clearly, this is not well justified; horizontal mixing has a diapycnal component and isopycnal mixing a vertical component when isopycnals slope. The assumption is made, nevertheless, because it allows for the simplest mixing parameterization; it will be demonstrated that in the results obtained here the approximation is quite good. Setting vertical mixing to zero over large regions of the ocean raises questions about numerical stability (e.g., Weaver and Sarachik 1990), which will be taken up in the discussion. Zero rather than small vertical mixing is applied because if one attempts to explore very small interior mixing, one might as well go all the way; small, finite values as observed in the open ocean are then assured to work under an otherwise identical setting. Moreover, vertical mixing effects can unambiguously be ascribed to boundary mixing.

If it can be established that no fundamental numerical instability arises, an interesting prospect opens up: Our theoretical picture of the ocean's deep circulation has been formed by the Stommel and Arons (Stommel et al. 1958; Stommel 1958, Stommel and Arons 1960a,b) assumption of uniform deep upwelling from the abyss, which, given horizontally constant stratification, is consistent with vertical advective-diffusive balance everywhere. ${ }^{1}$ By combining uniform upwelling with the planetary vorticity equation, the Stommel-Arons theory suggests a very definite horizontal flow pattern, with interior flow being eastward and poleward everywhere and equatorward flow occurring only in western boundary currents. How should the basic picture of deep flow look if no mixing occurred in the ocean interior? An

\footnotetext{
${ }^{1}$ Stommel and Arons (1960a) also analyzed flow patterns induced by nonuniform upwelling on a sphere. Horizontal dependence was smooth, however, unlike the sharp contrasts implied by pure boundary mixing. Moreover, the application of the theory to an idealized World Ocean (1960b) explicitly specified uniform upwelling.
}

attempt to answer this question provides the scientific focus for the modeling exercises reported here.

More specifically, it will be investigated how the strength and three-dimensional structure of the steadystate meridional overturning circulation are influenced by the boundary mixing, assuming that surface density is effectively prescribed as a simple function of latitude. Linking overturning strength to the surface density gradient through a theoretical model derived from first principles has eluded researchers so far. Welander (1971) derived a scaling relationship based on vertical advective-diffusive balance and on thermal-wind scaling for the zonal flow, which was postulated by Bryan (1987) to hold for the zonally averaged meridional flow as well. However, the thermal wind relationship links the latter to the density difference between eastern and western sidewalls. Whether the isotropy assumption is justified has not been established.

For simplicity, wind forcing is entirely neglected in this study. The wind stress strongly influences surface density and is hence important for a complete dynamical picture. However, Marotzke (1990) showed that if surface density was almost prescribed in an idealized steady-state GCM, the strength of the meridional overturning did not differ much between two runs with and without wind forcing, except very near the surface. Consistent with earlier studies (e.g., Stommel and Arons 1960a,b; Colin de Verdière 1988), only buoyancy forcing is admitted here.

This paper is organized as follows. Section 2 provides a very brief description of the numerical model used. Section 3 displays the salient model results, motivating the theoretical considerations of section 4. Section 5 contains a summary and discussion. ${ }^{2}$

\section{Model description}

The model used is the Geophysical Fluid Dynamics Laboratory (GFDL) primitive equation GCM, based on the method described in Bryan (1969), in the version documented and distributed by Cox (1984). The domain extends from the equator to $64^{\circ} \mathrm{N}$ and is $60^{\circ}$ wide; the bottom is at a constant depth of $4500 \mathrm{~m}$. Resolution is $3.75^{\circ}$ zonally by $4^{\circ}$ meridionally; there are 15 levels in the vertical varying smoothly in spacing from $50 \mathrm{~m}$ near the surface to $500 \mathrm{~m}$ at depth. The model is forced by restoring boundary conditions on both surface temperature and salinity; the target profiles are independent of longitude and vary like the cosine of latitude, with peakto-peak amplitudes of $27^{\circ} \mathrm{C}$ and $1.5 \mathrm{psu}$, respectively.

\footnotetext{
${ }^{2}$ While this manuscript was undergoing review, I received from R. Samelson (Woods Hole Oceanographic Institution) a preprint (Samelson 1997), in which he investigates the effects that enhanced mixing at the eastern boundary of an idealized ocean model has on the abyssal stratification. The foci of the two papers are thus very different.
} 
The restoring timescale is 30 days, which means that surface temperature and salinity, hence density, are very nearly prescribed. Surface wind stress is set to zero. Standard horizontal and vertical friction parameterizations are used with coefficients $2.5 \times 10^{5}$ and $10^{-2} \mathrm{~m}^{2}$ $\mathrm{s}^{-1}$, respectively; horizontal diffusivity is set to $10^{3} \mathrm{~m}^{2}$ $\mathrm{s}^{-1}$. Static instability is removed by a convective adjustment procedure in which the vertical diffusivity is increased to $1 \mathrm{~m}^{2} \mathrm{~s}^{-1}$; the vertical diffusion term is always evaluated implicitly. The configuration is thus nearly identical to the one used before in Marotzke (1991) and Weaver et al. (1993). Salinity as a second state variable and a nonlinear equation of state are retained so the experience from previous numerical experiments can be exploited, at the expense of making the comparison with the theoretical model (section 4) less straightforward.

The only nonstandard feature in this idealized model is the choice of vertical diffusivity under statically stable conditions. Apart from the columns adjacent to the side walls, vertical diffusivity is zero. This crude parameterization of boundary mixing is supposed to mimic the effect of a sloping lateral boundary, in the vicinity of which tidal mixing, for example, might be very strong. At this exploratory stage, neither the representation of boundary mixing nor that of diapycnal mixing in general are very sophisticated. In particular, simple horizontal eddy diffusion is employed; greater sophistication can be incorporated at a later stage if warranted.

\section{Experimental results}

\section{a. Steady-state runs}

All experiments start from an isothermal $\left(5^{\circ} \mathrm{C}\right)$ and isohaline (33 psu) ocean at rest; the experiments are run until near-equilibrium is reached, the criterion being a basin-averaged heat flux of less than $10^{-4} \mathrm{~W} \mathrm{~m}^{-2}$. In some experiments, a steady state could not be attained. The vertical mixing coefficient $k_{v}$ at the boundaries is varied between zero (no vertical mixing at all except through convective adjustment) and $100 \times 10^{-4} \mathrm{~m}^{2} \mathrm{~s}^{-1}$. With $k_{v}=0$, the model still loses heat at a rate of 0.3 $\mathrm{W} \mathrm{m}^{-2}$ after 8000 model years; while a steady state has not been reached, the model state at year 8000 will nevertheless be considered characteristic of this run. If $k_{v}$ is $30 \times 10^{-4} \mathrm{~m}^{2} \mathrm{~s}^{-1}$ or larger, a self-sustained oscillation ensues, which will be discussed below.

Figure 1 shows the meridional overturning streamfunction and the northward heat transport for a representative case, $k_{v}=5 \times 10^{-4} \mathrm{~m}^{2} \mathrm{~s}^{-1}$. The results are similar to those obtained previously with models using uniform vertical mixing and similar geometry and forcing (e.g., Bryan 1987; Weaver and Sarachik 1990; Marotzke 1990, 1991; Zhang et al. 1992; Weaver et al. 1993). Figure 1a displays a conventional, single overturning cell of $19.2 \mathrm{~Sv}$ maximum $\left(\mathrm{Sv} \equiv 10^{6} \mathrm{~m}^{3} \mathrm{~s}^{-1}\right)$. In this low-resolution model, almost one-quarter of all grid
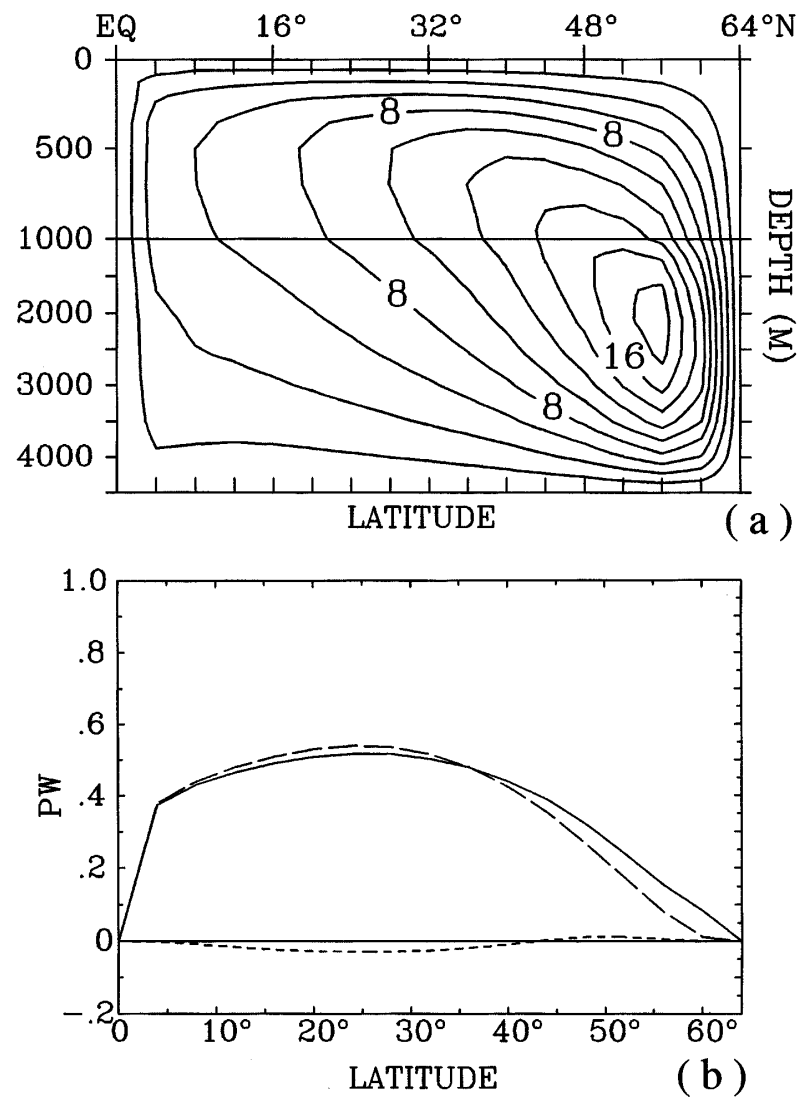

Fig. 1. Boundary mixing case, $k_{v}=5 \times 10^{-4} \mathrm{~m}^{2} \mathrm{~s}^{-1}$. (a) Meridional overturning, contour interval $2 \mathrm{~Sv}$. The flow is such that high values are to the right, that is, clockwise around a high and anticlockwise around a low. (b) Meridional heat transport; solid: total, long-dashed: overturning contribution (vertical correlation of zonally averaged temperature and velocity), and short-dashed: gyre contribution (correlation of deviations from zonal mean).

points are boundary points ( 60 out of 256 ), so the areaaveraged diffusivity is $0.9 \times 10^{-4} \mathrm{~m}^{2} \mathrm{~s}^{-1}$, close to Munk's (1966) estimate. The overturning is slightly stronger than the one Bryan (1987) obtained with uniform vertical diffusivity of $10^{-4} \mathrm{~m}^{2} \mathrm{~s}^{-1}$; the comparison with the uniform mixing case will be taken up below. Northward heat transport in our results is about $0.5 \mathrm{PW}$ $\left(1 \mathrm{PW} \equiv 10^{15}\right.$ watts, Fig. 1b) at its maximum; most of the transport is carried by the overturning contribution (i.e., the vertical correlation of zonally averaged temperature and velocity).

A summary of the runs in which a steady state is found is given in Fig. 2, showing maximum meridional overturning of the whole basin and maximum meridional overturning at $32^{\circ} \mathrm{N}$, both taken to the $3 / 2$ power, and squared maximum northward heat transport, all as functions of $k_{v}$. These particular powers of the physical quantities were chosen for representation following some experimentation; previous scaling analyses and numerical solutions (e.g., Bryan 1987; Huang and Chou 1994) have suggested a power law dependence on vertical diffusivity. All three quantities displayed in Fig. 2 

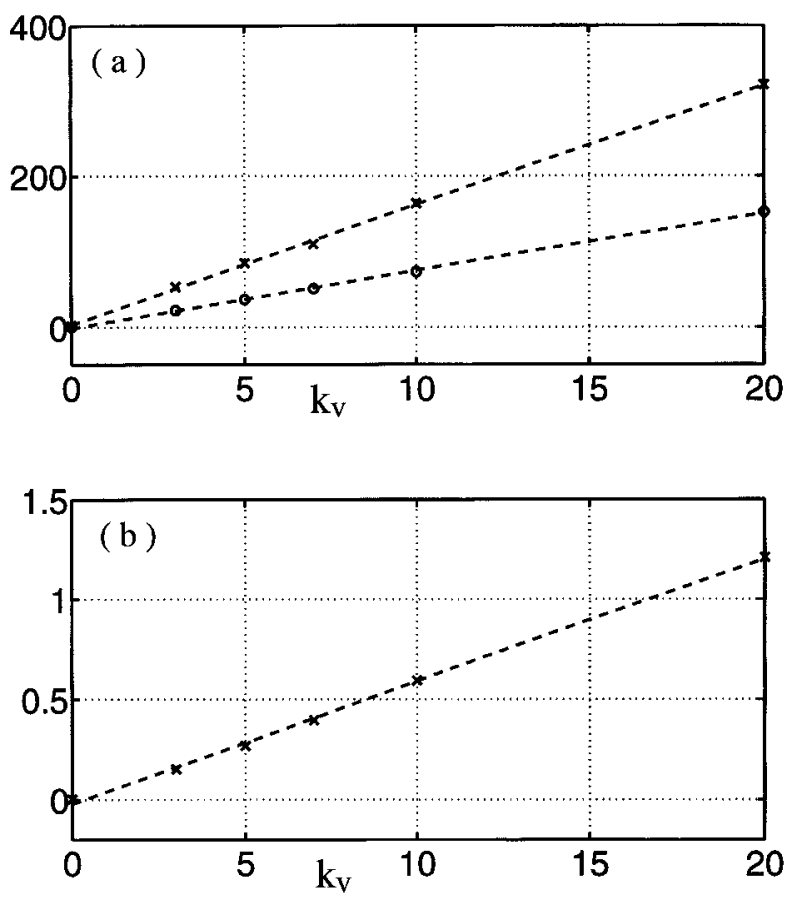

FIG. 2. (a) Maximum value of the overturning streamfunction over the entire basin (crosses) and at $32^{\circ} \mathrm{N}$ (circles) as a function of vertical mixing near the side boundaries. The overturning values are taken to the $3 / 2$ power; the straight lines are least squares fits and indicate a $2 / 3$ power dependence of overturning strength on $k_{v}$. (b) Squared maximum heat transport as a function of vertical mixing near the side boundaries; the fit indicates a 1/2 power dependence of heat transport on $k_{v}$.

follow a power law remarkably well, overturning being proportional to $k_{v}^{2 / 3}$, and heat transport proportional to $k_{v}^{1 / 2}$. A derivation of these power laws is given in section 4 below; for the moment, we will focus on the threedimensional numerical solution structure.

Figure 3 shows vertical velocity at $34^{\circ} \mathrm{N}$, the western wall, and the eastern wall. At a typical midlatitude zonal section, vertical motion is mostly confined to the column adjacent to the boundary (these experiments were run without wind forcing so there is no wind-induced vertical motion in the ocean interior). Interior vertical motion is confined to the vicinity of the boundary regions and is smaller by an order of magnitude. At the western wall, there is upwelling only, while in the east there is downwelling to a depth of about $500 \mathrm{~m}$ with upwelling underneath. Figure $3 \mathrm{~b}$ demonstrates that vertical motion is upward along the entire western boundary, with strength increasing toward higher latitudes. At the eastern wall, the vertical location of zero vertical motion decreases from zero at the equator to the bottom at $54^{\circ} \mathrm{N}$, north of which downwelling prevails at all depths.

The change in sign in eastern boundary vertical motion means that the pattern is more complex than the qualitative picture of meridional overturning force balance depicted, for example, by Zhang et al. (1992) and Colin de Verdière (1993), who stipulated that in re-
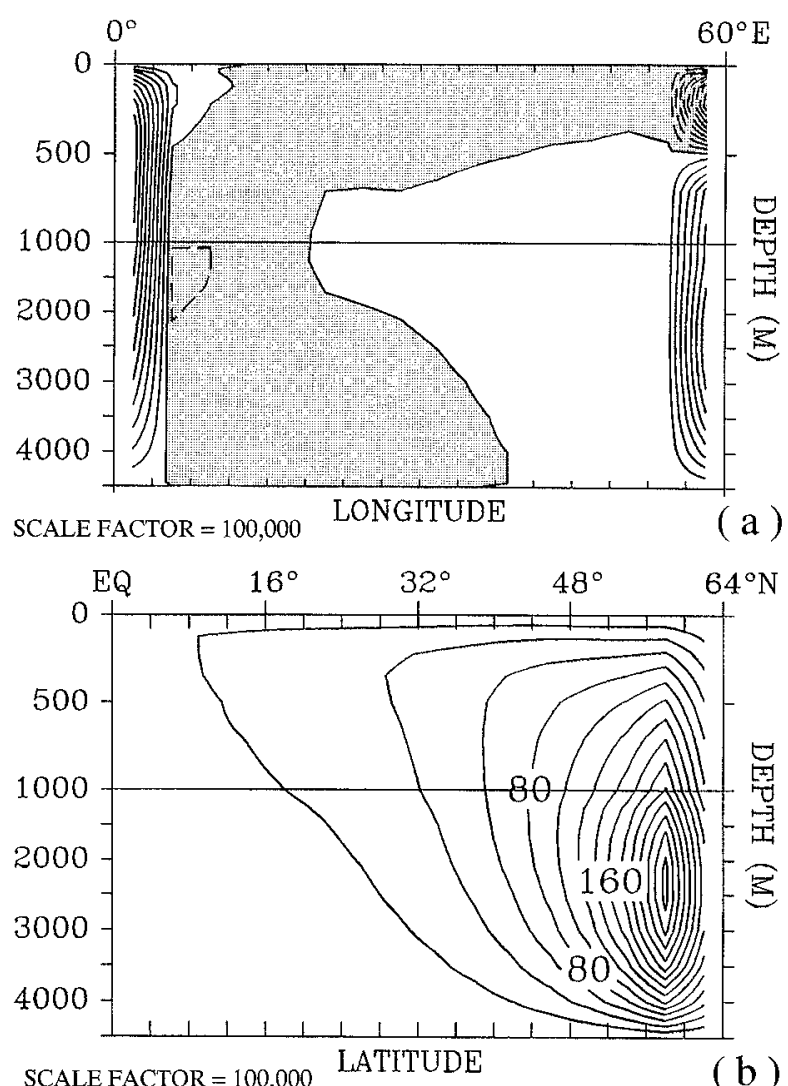

SCALE FACTOR $=100,000 \quad$ LATTTUDE

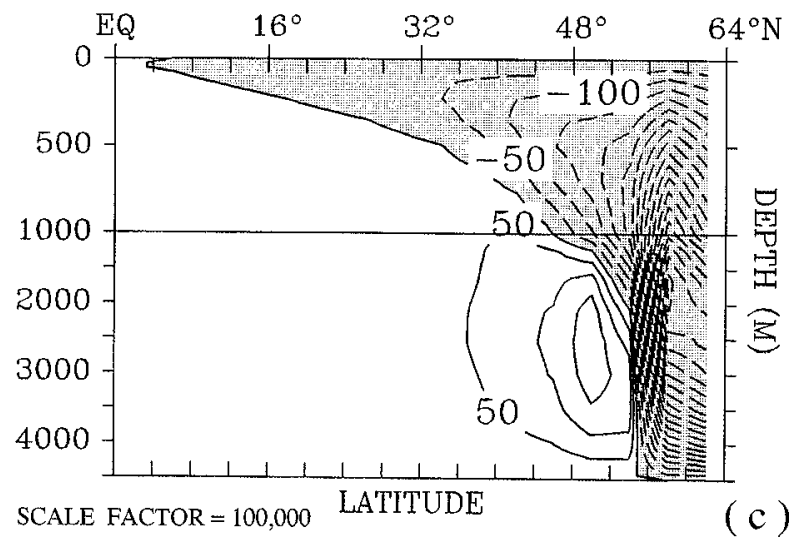

FIG. 3. Vertical velocity for the boundary mixing case, $k_{v}=5 \times$ $10^{-4} \mathrm{~m}^{2} \mathrm{~s}^{-1}$, (a) along $34^{\circ} \mathrm{N}$, contour interval $5 \times 10^{-5} \mathrm{~cm} \mathrm{~s}^{-1}$; (b) along the western boundary, contour interval $20 \times 10^{-5} \mathrm{~cm} \mathrm{~s}^{-1}$; and (c) along the eastern boundary, contour interval $50 \times 10^{-5} \mathrm{~cm} \mathrm{~s}^{-1}$. Areas of downwelling are marked by dashed contours and shading. Notice that all velocity values are scaled by $10^{5}$; the numbers shown correspond roughly to centimeters per day.

sponse to a meridional surface density gradient, eastward surface flow would ensue, impinge on the eastern boundary, sink, and return westward at depth. The result would be lower densities at the eastern than at the western wall; hence northward zonal-mean shear and the traditional overturning picture would emerge. Winton (1996) obtained vertical motion near the eastern bound- 
ary very similar to Fig. 3c, in a planetary-geostrophic $f$-plane model with uniform vertical diffusion; he called the downwelling over upwelling circulation surprising, probably in light of the simpler qualitative picture. In section 4, I will argue that this circulation pattern must emerge as a very robust feature in the numerical experiments presented here.

The different vertical velocity patterns between eastern and western boundaries are reflected in the temperature sections along the walls (Fig. 4). In the west, the water is stratified everywhere except right at the northern boundary, and stratification is strongest at the surface. In the east, convection leads to neutral stratification to a depth that increases with latitude, and maximum stratification occurs roughly where vertical velocity changes sign (i.e., at the depth of maximum vertical convergence; compare Fig. 3c). Figure 4c displays the density difference between eastern and western walls, a measure of the vertical shear of zonally averaged meridional velocity. Moving from the equator poleward, the density difference becomes stronger and extends to greater depths, enhancing shear and strength of the meridional circulation; north of roughly $48^{\circ} \mathrm{N}$ the shear becomes weaker again since the mean zonal density difference decreases.

Figure $4 \mathrm{~b}$ shows that, even in the absence of windinduced equatorial upwelling, middepth isotherms (and hence isopycnals) slope downward with latitude at the eastern boundary. This density structure leads to westward shear consistent with the need to provide a pathway for the vertically converging waters (Fig. 3c). Figure $5 \mathrm{a}$, showing zonal flow at $36^{\circ} \mathrm{N}$, makes this point explicit. It shows that zonal flow is layered, with eastward surface currents overlying westward middepth flow, itself above weak eastward deep currents. Meridional velocity at the same latitude (Fig. 5b) shows that the mass balance at the eastern boundary is purely between zonal and vertical flows; vigorous meridional flow is confined to the western boundary with the traditional two-layered pattern; there are indications of Munk-type southward recirculation just east of the "Gulf Stream." Consistent with Fig. 5b, the zonal temperature section along $34^{\circ} \mathrm{N}$ shows significant slope only at the western boundary, apart from some near-surface structure (Fig. 5c). Indeed, one would expect eastern boundary current structures to be eroded by Rossby wave radiation.

Horizontal velocity in the surface and bottom layers are displayed in Fig. 6. Surface flow (Fig. 6a) is almost entirely zonal, with the exception of the western boundary current and the aforementioned Munk-type recirculation. In the abyss, the Stommel-Arons theory suggests a very definite horizontal flow pattern, with interior flow being poleward everywhere and equatorward flow occurring only in western boundary currents. With boundary mixing only, the numerical results look entirely different (Fig. 6). Nonvanishing meridional flow is found, broadly speaking, only in the convection
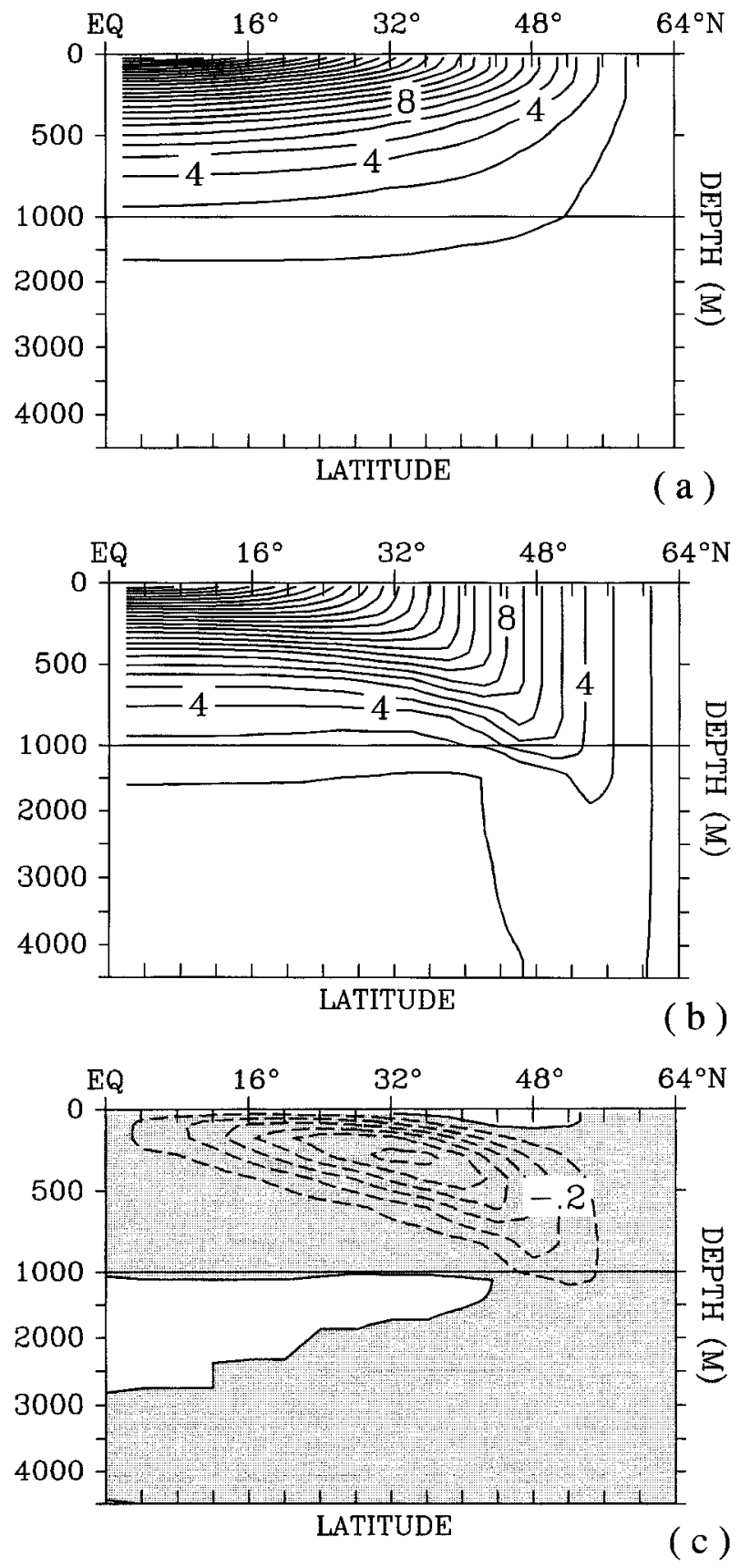

FIG. 4. Boundary mixing case, $k_{v}=5 \times 10^{-4} \mathrm{~m}^{2} \mathrm{~s}^{-1}$. (a) Temperature along the western boundary, contour interval $1^{\circ} \mathrm{C}$. (b) Temperature along the eastern boundary, contour interval $1^{\circ} \mathrm{C}$. (c) Potential density difference as eastern wall minus western wall, contour interval $0.1 \mathrm{~kg} \mathrm{~m}^{-3}$. Areas with negative values are marked by dashed contours and shading.

regions and the western boundary. Consistent with very weak interior vertical motion and a balance between vortex stretching and planetary vorticity advection, interior flow is zonal. By mass continuity, one can infer from Fig. $6 \mathrm{~b}$ that deep upwelling occurs at the eastern 

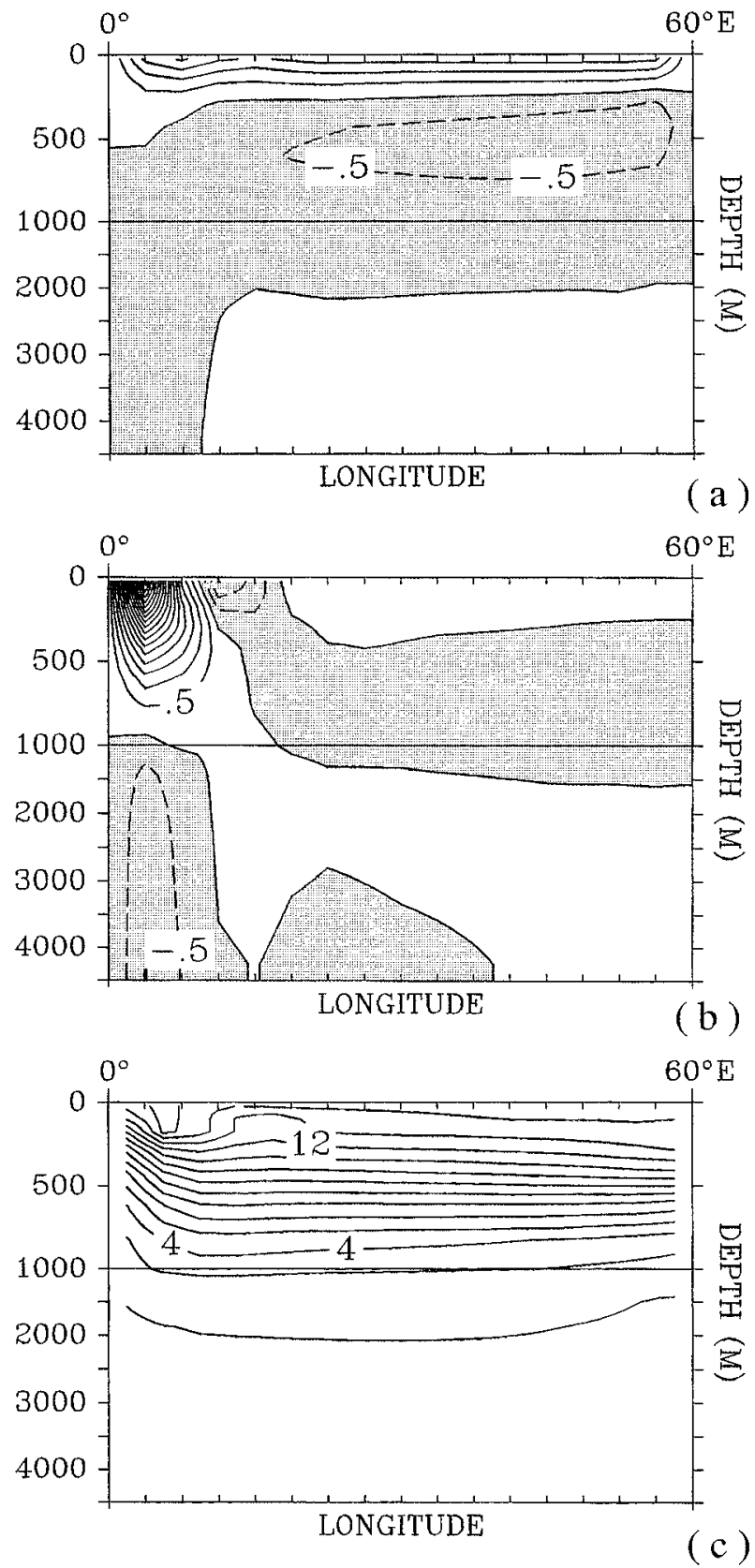

FIG. 5. Boundary mixing case, $k_{v}=5 \times 10^{-4} \mathrm{~m}^{2} \mathrm{~s}^{-1}$. (a) Zonal velocity along $36^{\circ} \mathrm{N}$ and (b) meridional velocity across $36^{\circ} \mathrm{N}$, contour interval $0.5 \mathrm{~cm} \mathrm{~s}^{-1}$. (c) Temperature along $36^{\circ} \mathrm{N}$, contour interval $1{ }^{\circ} \mathrm{C}$. Areas with negative values are marked by dashed contours and shading.

and western boundaries, and downwelling in the northeastern corner.

\section{b. Comparison with uniform diffusivity}

Figure 7 displays a few solution features of a run with uniform vertical diffusion of $0.9 \times 10^{-4} \mathrm{~m}^{2} \mathrm{~s}^{-1}$, that is, the area-averaged diffusivity is the same as in Figs. 1 and 3-6. All other run parameters are identical. With uniform vertical diffusion, maximum overturning strength is weaker by $18 \%$ (15.7 Sv in Fig. 7a compared to $19.2 \mathrm{~Sv}$ in Fig. 1a); maximum heat transport is weaker by $25 \%$ ( $0.39 \mathrm{PW}$ in Fig. $7 \mathrm{~b}$ compared to $0.52 \mathrm{PW}$ in Fig. 1b). Vertical velocity at $34^{\circ} \mathrm{N}$ (Fig. 7c) is quite similar to the boundary mixing case in and near the western boundary (Fig. 3a). Also, the near-surface downwelling at the eastern boundary is similar in strength and penetration depth. However, the upwelling at the eastern boundary is much weaker and less coherent, and interior upwelling is stronger by a factor of 2 or more. Horizontal flow (not shown) is rather similar to that of the boundary mixing case (Fig. 6); near the eastern boundary, there is some southward flow in the thermocline and some northward flow at depth, both counteracting the western boundary current at their respective depths and hence weakening the meridional overturning.

The uniform mixing case does not exhibit the simple Stommel-Arons picture of uniform interior upwelling and the resulting horizontal flow pattern, which is consistent with the results of Huang and Chou (1994) and the recent analysis by T. Huck and A. J. Weaver (1996, personal communication). Whether the solution should show the Stommel-Arons picture of uniform upwelling is another matter, having to do with the question of whether the western boundary upwelling is considered an effect of spurious horizontal, diapycnal mixing or of "real" vertical mixing. Due to their exploratory nature, the runs presented here employ horizontal diffusion for simplicity and are thus open to criticism for not controlling diapycnal mixing rigorously enough. Figure $3 \mathrm{a}$ shows, however, that in the boundary mixing case the overwhelming part of the diapycnal mixing occurs through vertical mixing near the walls. One can then conclude from Fig. $7 \mathrm{c}$ that even in the uniform mixing case, the majority of the mixing occurs near the boundary, but that "leakage" occurs (i.e., interior upwelling). If the rotating dynamics all by themselves try to concentrate vertical motion near the boundaries (e.g., Huang and Chou 1994; Winton 1996), one does not expect the Stommel-Arons picture to hold particularly well. In addition, any kind of scaling relationship is likely to work better if mixing is entirely confined to the boundaries, thus excluding the leakage of upwelling.

\section{c. Self-sustained oscillations}

Under the same boundary conditions as hitherto employed, but with boundary diffusivities of $30 \times 10^{-4}$ $\mathrm{m}^{2} \mathrm{~s}^{-1}$ and higher (no interior vertical mixing), no steady-state solutions can be found; instead, self-sustained oscillations on a roughly 25 -yr timescale ensue. Figure 8 shows meridional overturning near the extremes of the oscillation for $k_{v}=40 \times 10^{-4} \mathrm{~m}^{2} \mathrm{~s}^{-1}$. Total high-latitude downwelling only varies between 70 Sv (Fig. 8a) and $85 \mathrm{~Sv}$ (Fig. 8b) for the "weak" and 


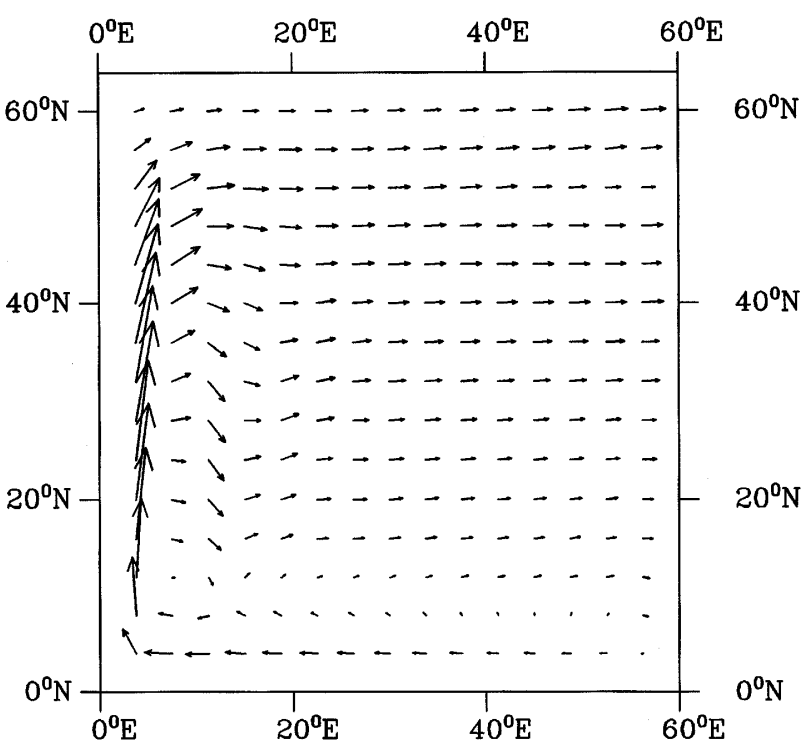

( a )

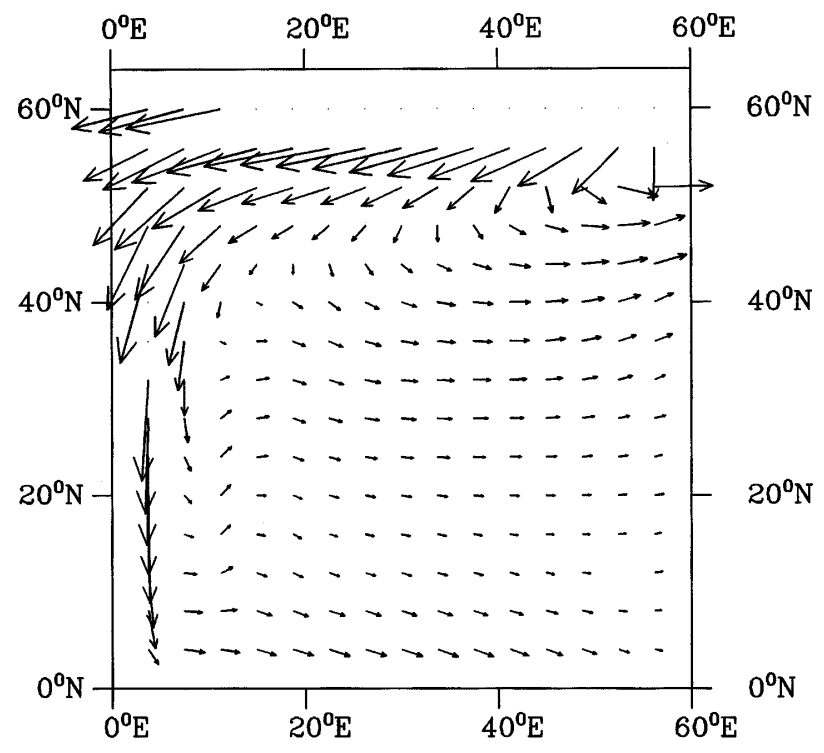

(b)

FIG. 6. Boundary mixing case, $k_{v}=5 \times 10^{-4} \mathrm{~m}^{2} \mathrm{~s}^{-1}$. (a) Horizontal velocity at 25-m depth (first model level), reference vector $10 \mathrm{~cm}$ $\mathrm{s}^{-1}$ and (b) horizontal velocity at 4250-m depth (lowest model level), reference vector $1 \mathrm{~cm} \mathrm{~s}^{-1}$; no vectors are shown where the speed exceeds $1 \mathrm{~cm} \mathrm{~s}^{-1}$.

"strong" phases of the circulation, respectively, but during the strong phase the overturning consists of a single cell, whereas during the weak phase more than half of the sinking water turns northward and upwells right at the northern boundary. This pulsation of a thermally indirect cell, emanating from the northern boundary and retreating, was previously described by Marotzke (1990) as occurring during a "flush" under mixed thermohaline boundary conditions (restoring condition for surface temperature; flux condition for surface salinity). During a flush, the model deep ocean within 100 years lost a large amount of heat that it had accumulated during several thousand years of a basinwide thermally indirect circulation. There, the oscillation was a transient phenomenon occurring during the flush. Here, the oscillations persist, indicating that their fundamental cause lies not in the flush or the mixed boundary conditions, but in convection reaching to the bottom all across the high latitudes.

A direct mean meridional circulation cell is supported dynamically by lower densities at the eastern wall than at the western wall, a pattern which in turn is supported by the vigorous upwelling at the western boundary (Figs. 3 and 4). As the vertical mixing coefficient is increased, meridional overturning strength, poleward heat transport, and convective activity increase as well. If an entire latitude strip is convecting, the stratification is neutral everywhere, and upwelling at the western wall can no longer lead to higher-density water there than at the eastern wall. Rather, one expects the western region to be warmer (hence lighter) than the eastern boundary, since south of the convecting latitude the western boundary current leads to the highest temperatures in the west. Resulting from the "reversed" mean zonal density gradient (see Fig. 9a) is a reversed meridional overturning circulation (Fig. 8a), which however leads to its own demise. The poleward near-surface boundary current is weak at high latitudes (Fig. 9b), as is heat transport, and the "warm pool" near the western wall is cooled until temperatures are nearly zonally uniform again. Then, the heating from the south leads to a local maximum in temperature near to, but detached from, the western boundary. Convection is interrupted there, a boundary current builds up that transports heat northward, and for a short while a strongly stratified strip of upwelling exists inshore of the boundary current (Fig. 9c), which can support a strong boundary current (Fig. 9d) and a thermally direct overturning cell reaching to the northern wall (Fig. 8b). Finally, the heat transport leads to too strong heating of the western boundary, the overturning circulation reverses, and the cycle starts over again.

A detailed account of this oscillation is beyond the scope of this paper, but a very general dynamical argument emerges for the sense of the overturning and the existence of a steady state: The presence (absence) of strongly stratified water near the western boundary depends on convection occurring only in the eastern portion (everywhere along a latitude circle) and leads to thermally direct (indirect) meridional overturning. The indirect overturning cannot attain a steady state because it cannot provide the heat transport necessary to keep the western region warm enough; if temporarily a direct circulation ensues, its heat transport is too strong to keep the western boundary columns cold enough. Weaver et al. (1994) found oscillations in the Labrador 


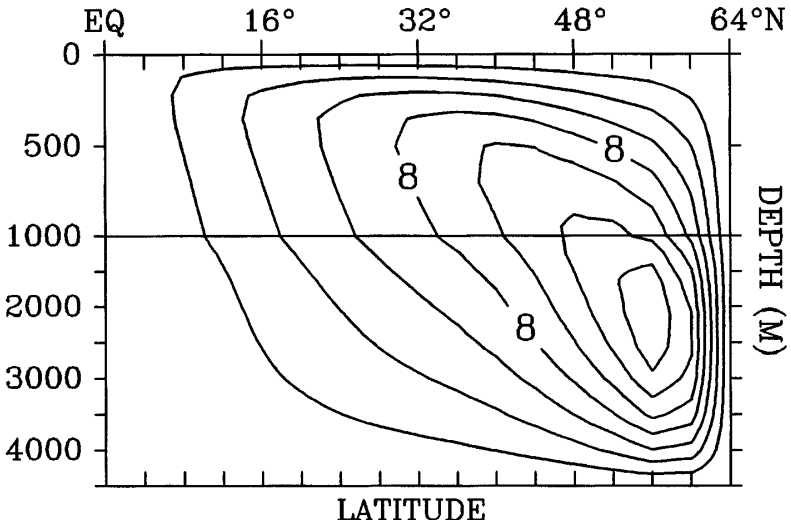

( a )
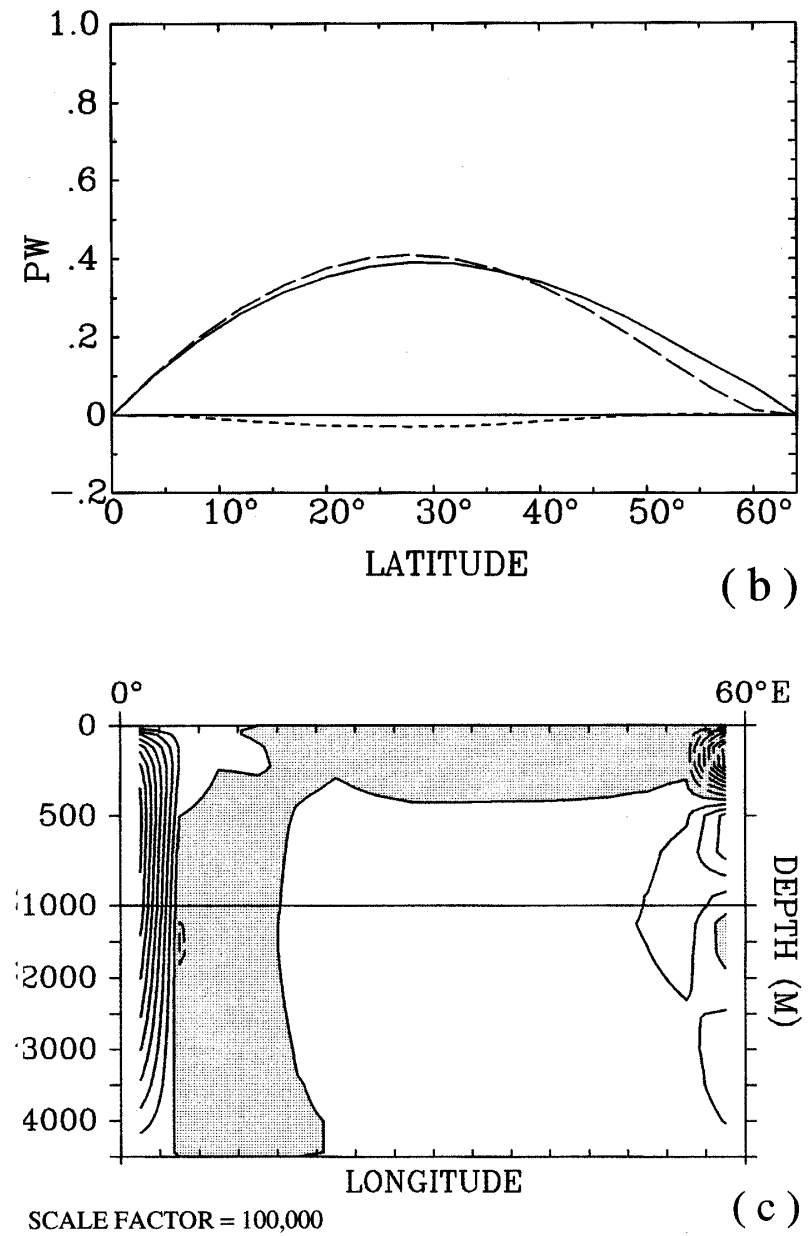

FIG. 7. Uniform mixing case, $k_{v}=0.9 \times 10^{-4} \mathrm{~m}^{2} \mathrm{~s}^{-1}$. (a) Meridional overturning, contour interval $2 \mathrm{~Sv}$. The flow is such that high values are to the right, that is, clockwise around a high and anticlockwise around a low. (b) Meridional heat transport; solid: total, long-dashed: overturning contribution (vertical correlation of zonally averaged temperature and velocity), and short-dashed: gyre contribution (correlation of deviations from zonal mean). (c) Vertical velocity along $34^{\circ} \mathrm{N}$, contour interval $5 \times 10^{-5} \mathrm{~cm} \mathrm{~s}^{-1}$ scaled by $10^{5}$; (the numbers shown correspond roughly to $\mathrm{cm} /$ day). Areas of downwelling are marked by dashed contours and shading.

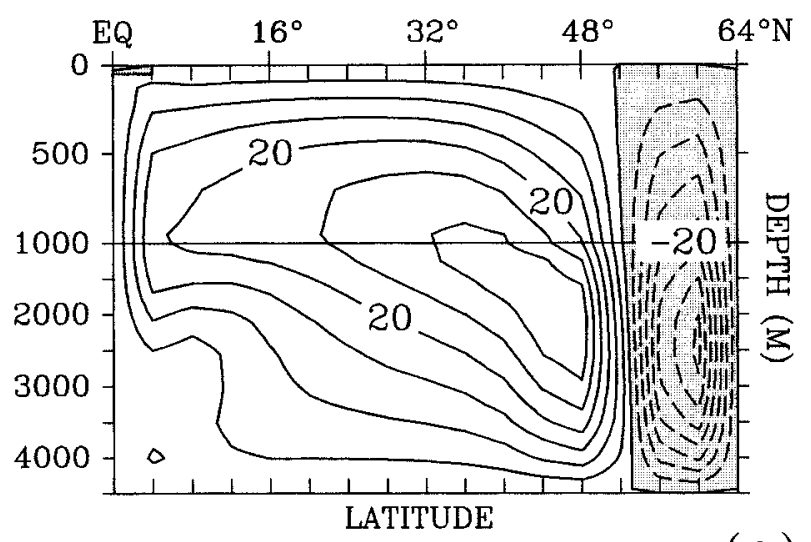

(a)

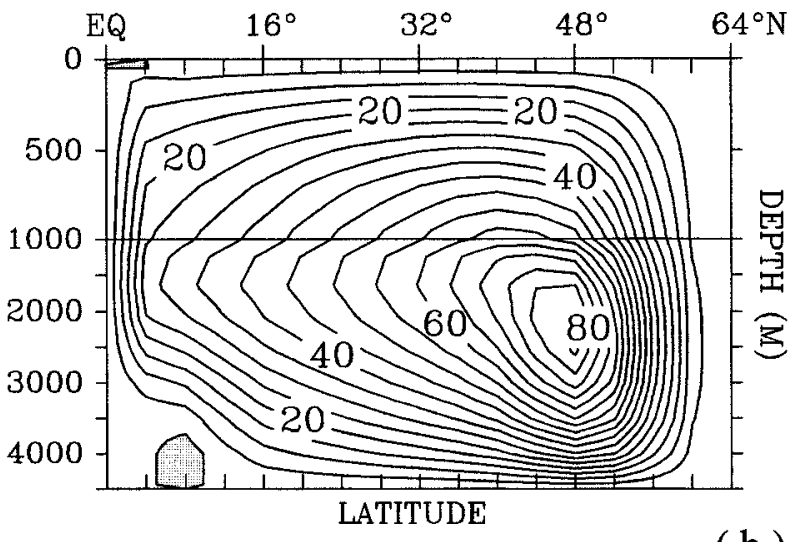

(b)

FIG. 8. Boundary mixing case, $k_{v}=40 \times 10^{-4} \mathrm{~m}^{2} \mathrm{~s}^{-1}$. Meridional overturning during (a) weak $(t=0)$ and (b) strong phases $(t=15$ yr) of the direct circulation, contour interval $5 \mathrm{~Sv}$. Negative contours are dashed, and areas with negative values are shaded. The flow is such that high values are to the right, that is, clockwise around a high and anticlockwise around a low.

Sea portion of their idealized North Atlantic model similar in appearance and dynamics to the one described here (although an indirect cell did not appear), and demonstrated their existence even when they used purely restoring thermodynamic boundary conditions and no wind forcing. Similarly, Fanning and Weaver (1997) found solutions with a reverse cell waxing and waning on a 15-yr timescale, in a GCM geometry similar to the one here, but with uniform vertical mixing, higher resolution, and under various stages of coupling to the atmosphere.

\section{Theory}

It was indicated earlier on that, since even the uniform mixing case leads to vertical motion more confined to the lateral boundaries, it might be simpler to analyze the three-dimensional dynamics of the thermohaline circulation in the boundary mixing case. This section will derive a scaling relationship for the meridional overturning strength, by directly estimating the mean zonal 

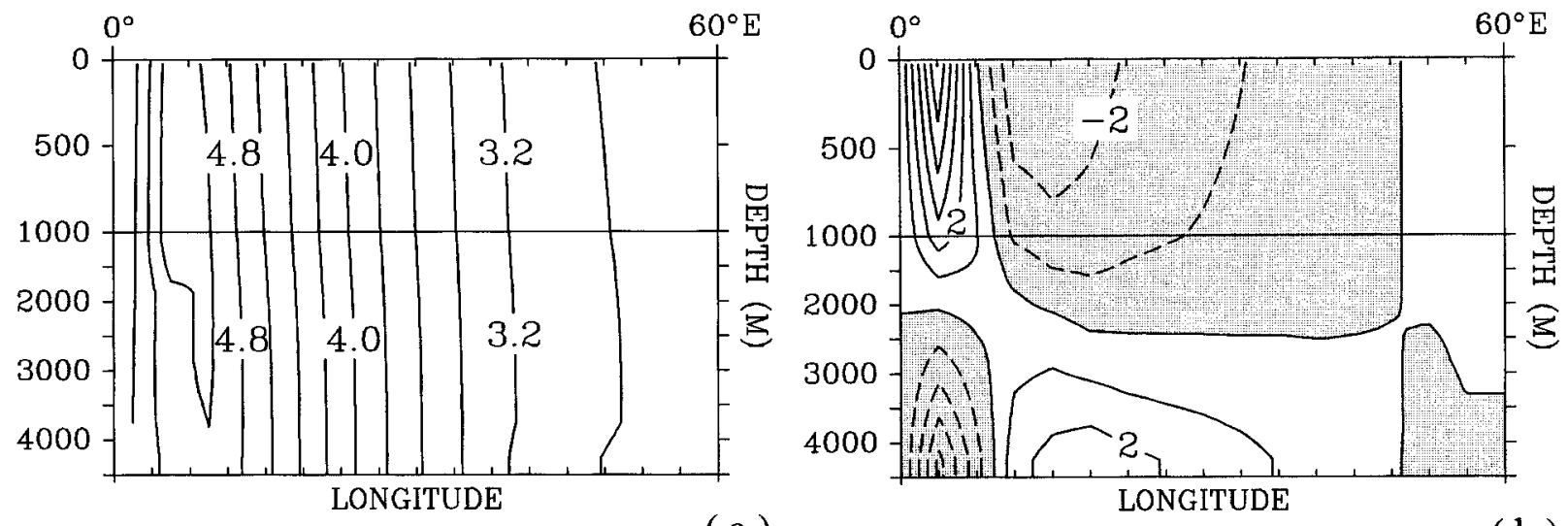

(a)

( b )
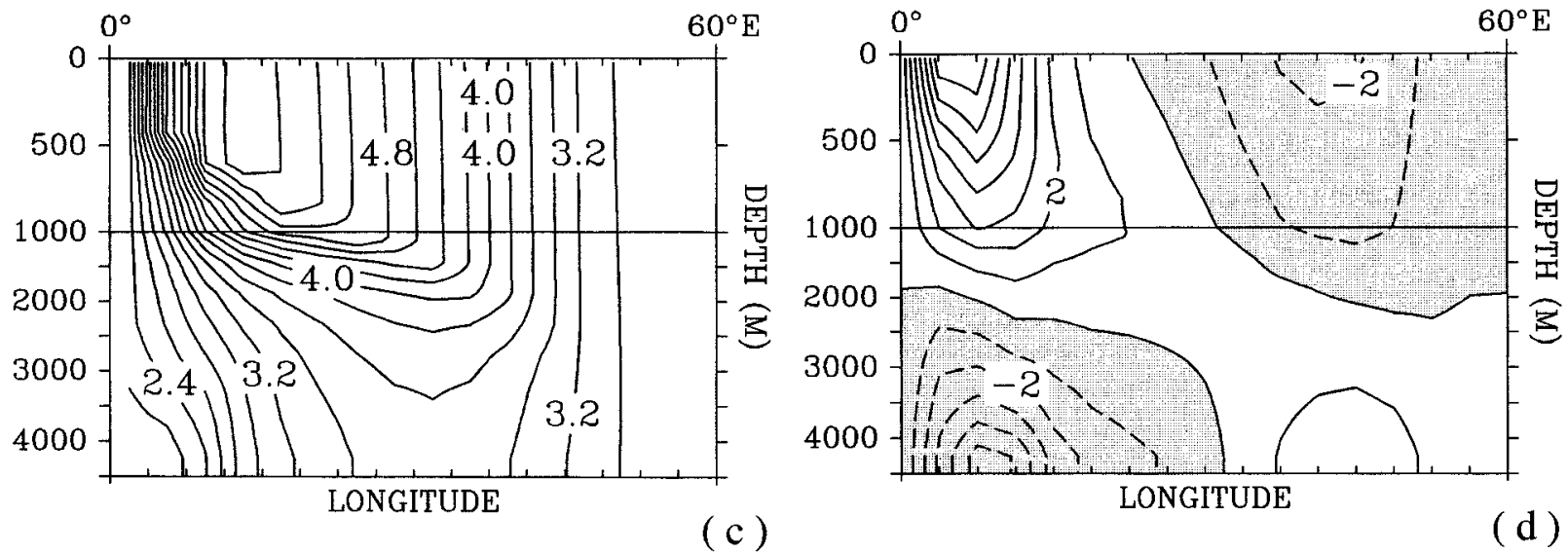

FIG. 9. Boundary mixing case, $k_{v}=40 \times 10^{-4} \mathrm{~m}^{2} \mathrm{~s}^{-1}$. (a) Temperature along $58^{\circ} \mathrm{N}$ during weak phase $(t=0)$ of the direct circulation, contour interval $0.2^{\circ} \mathrm{C}$. (b) Meridional velocity across $56^{\circ} \mathrm{N}$ during weak phase $(t=0)$, contour interval $1 \mathrm{~cm} \mathrm{~s}^{-1}$. Southward flow is characterized by dashed contours and shading. (c) Temperature along $58^{\circ} \mathrm{N}$ during strong phase $(t=15 \mathrm{yr})$, contour interval $0.2^{\circ} \mathrm{C}$. (d) Meridional velocity across $56^{\circ} \mathrm{N}$ during strong phase $(t=15 \mathrm{yr})$, contour interval $1 \mathrm{~cm} \mathrm{~s}^{-1}$. Southward flow is marked by dashed contours and shading.

density gradient from the independent parameters of the problem.

Four fundamental assumptions are made, in addition to the standard approximations (hydrostatic and geostrophic balance):

1) Surface density is given and is a function of latitude only; the abyss uniformly has the properties of the densest surface water.

2) The western boundary water is assumed to be stably stratified, following an exponential with scale height $D$ (to be determined as part of the solution).

3) Density in the grid cells at the lateral boundary is governed by vertical advective-diffusive balance,

$$
w \partial_{z} \rho=k_{v} \partial_{z z} \rho
$$

except where convection is present, which then also enters the balance.

4) Along the eastern boundary, convection occurs down to a depth $z_{\rho}$ (to be determined as part of the solution), which is a function of latitude; in other words, the isopycnal $\rho=$ const. is vertical at its outcrop latitude. Equatorward, it is assumed level; likewise, it is assumed that Rossby wave activity has eliminated all zonal isopycnal slopes except in the western boundary current.

Figure 10 illustrates the quantities introduced here. Assumption (1) was used before by Welander (1971) and Bryan (1987), (2) is a standard assumption, and (3) is familiar in its uniformly valid version, which underlies Bryan's (1987) scaling and indirectly the StommelArons picture. Here, it is well justified since vertical mixing near the boundary is strong. Assumption (4) is probably the most unorthodox; it is based on the physical picture that warm water generally moves to the northeast; subsurface advection of a certain density can only occur until the outcrop latitude is reached. Assuming isopycnals either vertical or level at the eastern boundary is inconsistent with the model results (Fig. 4b) shown above; in the absence of isopycnals sloping downward with latitude, the "mode-2" structure in zonal flow cannot be supported. This feature, while qualitatively and quantitatively important (see below), will 


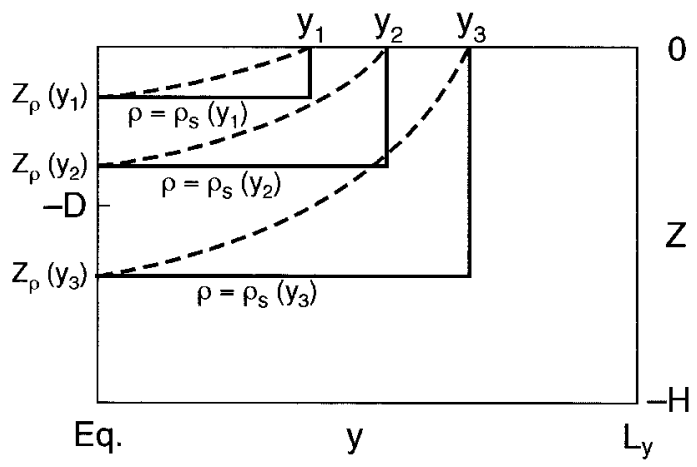

FIG. 10. Sketch of the analytical model (latitude-depth section). Shown are sample isopycnals at the eastern (solid) and western walls (dashed); notice that the model is continuous. Between east and west, there is no difference in outcrop latitudes or height $\left(z_{\rho}\right)$ at the equator The depth $D$ is the exponential scale height in the western boundary.

nevertheless be ignored since its inclusion would complicate the procedure considerably.

As discussed later, assumptions (iii) and (iv) are not compatible over a certain depth range below the mixed layer at the eastern boundary and thus are not adopted exactly in the following. Instead, they will serve as guides in obtaining approximate expressions for the zonal density difference and the meridional overturning streamfunction. The most important point about assumption (iv) is that convection is an integral part of the theoretical framework, in accord with the results of Zhang et al. (1992) who, in their planetary-geostrophic model, found that the meridional overturning changed from a thermally direct circulation to a thermally indirect one (with sinking at low latitudes) when they turned off their convective adjustment algorithm. The assumption of no interior zonal isopycnal tilt has previously been made by Sakai and Peltier (1995) in their two-dimensional closure of the three-dimensional thermohaline circulation.

To simplify matters, the surface density $\rho_{S}$ is assumed to be a linear function of latitude,

$$
\rho_{S}(y)=\rho_{P}+\left(\rho_{T}-\rho_{P}\right)\left(1-y / L_{y}\right),
$$

where $y$ is latitude, $L_{y}$ is meridional extent, and $\rho_{T}$ and $\rho_{P}$ are surface densities at the southern (tropical) and northern (polar) walls respectively. Near the western wall density is approximately, according to assumption (ii) and Eq. (2),

$$
\rho_{W}(y, z)=\rho_{P}+\left(\rho_{T}-\rho_{P}\right)\left(1-y / L_{y}\right) \exp (z / D) .
$$

Equation (3) is an approximation since it assumes that $D \ll H$, where $H$ is total depth, for the deep density to be equal to $\rho_{P}$. Define $z_{\rho}$ as the depth (strictly speaking, height, since $z$ is negative) of isopycnal $\rho$ at the equator,

$$
\rho\left(y=0, z_{\rho}\right) \equiv \rho_{P}+\left(\rho_{T}-\rho_{P}\right) \exp \left(z_{\rho} / D\right) .
$$

Since there is no wind stress in this model, no zonal pressure gradient can be supported at the equator, and $z_{\rho}$ is independent of longitude. Denote with $y_{\text {out }}$ the lat-
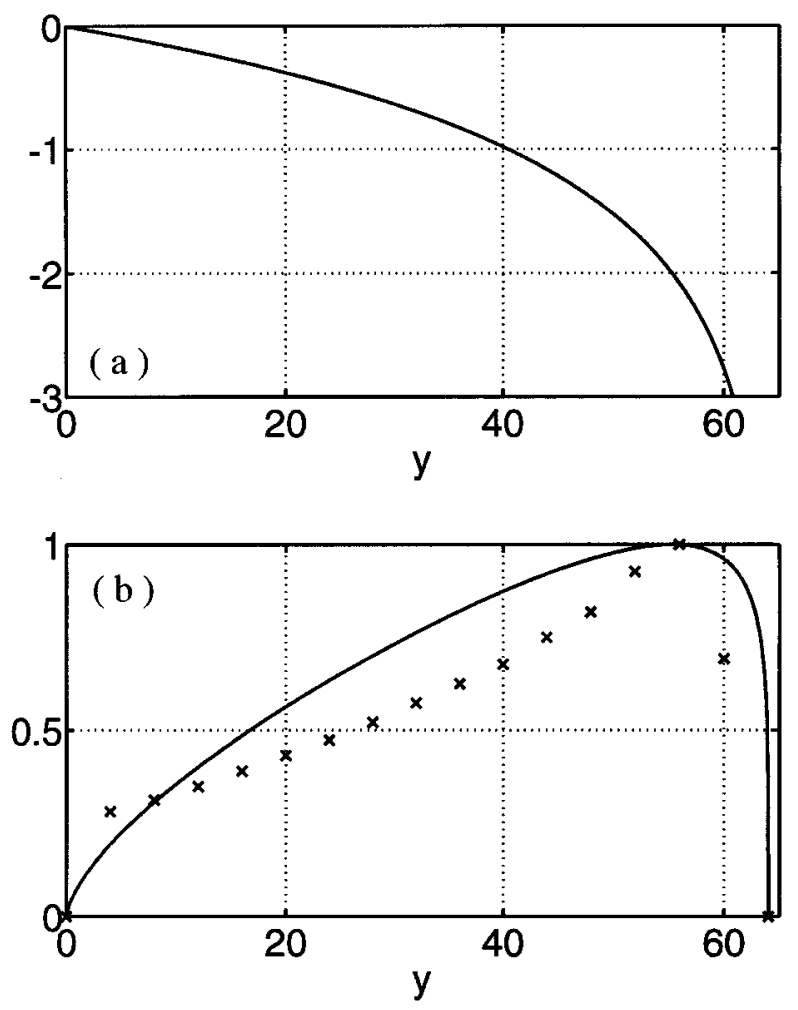

FIG. 11. (a) (Negative) depth of convection along the eastern boundary $z_{\rho}$ divided by "thermocline depth" $D$, according to scaling [Eq. (6)]. (b) Latitudinal dependence of overturning according to scaling [Eq. (13), solid] and from the numerical experiment (maximum strength at every latitudinal grid point, see Fig. 1a; boundary mixing case, $k_{v}=5 \times 10^{-4} \mathrm{~m}^{2} \mathrm{~s}^{-1}$; crosses).

itude at which isopycnal $\rho$ outcrops; equatorial density at depth $z_{\rho}$ is then equal to surface density at latitude $y_{\text {out }}$. Outcrop latitude and equatorial depth of an arbitrary isopycnal are thus linked by equating Eqs. (2) and (4);

$$
\begin{aligned}
\rho_{P} & +\left(\rho_{T}-\rho_{P}\right)\left(1-y_{\text {out }} / L_{y}\right) \\
& =\rho_{P}+\left(\rho_{T}-\rho_{P}\right) \exp \left(z_{\rho} / D\right),
\end{aligned}
$$

which gives

$$
z_{\rho}=D \ln \left(1-\frac{y_{\text {out }}}{L_{y}}\right)
$$

Notice that $z_{\rho}$ is negative since the argument of the natural logarithm is less than one. Along the eastern boundary, assumption (iv) states that the depth of the isopycnal does not change between the equator and the outcropping latitude. Hence, Eq. (6) also gives the depth of convection along the eastern boundary, going from zero at the equator $(y=0)$ to $D$ at $y=0.63 L_{y}$ to the bottom (literally to infinite depth) at $y=L_{y}$ (see Fig. 11a).

Density along the eastern boundary is, hence, 


$$
\begin{gathered}
\rho_{E}(y, z)=\rho_{S}(y)=\rho_{P}+\left(\rho_{T}-\rho_{P}\right)\left(1-y / L_{y}\right), \\
z>z_{\rho} \\
\rho_{E}(y, z)=\rho(0, z)=\rho_{P}+\left(\rho_{T}-\rho_{P}\right) \exp (z / D), \\
z<z_{\rho} .
\end{gathered}
$$

The density difference between eastern and western walls now follows as

$$
\begin{aligned}
& \rho_{E}(y, z)-\rho_{W}(y, z) \\
& =\left(\rho_{T}-\rho_{P}\right)\left(1-y / L_{y}\right)[1-\exp (z / D)], \quad z>z_{\rho} \\
& \rho_{E}(y, z)-\rho_{W}(y, z) \\
& =\left(\rho_{T}-\rho_{P}\right) y / L_{y} \exp (z / D), \\
& z<z_{\rho} \text {. }
\end{aligned}
$$

The absolute magnitude of this difference decreases with latitude above $z_{\rho}$ and increases with latitude below $z_{\rho}$ (which itself is a function of latitude), qualitatively in accord with the numerical solution shown in Fig. 4c. It is readily shown that the absolute magnitude of the east-west density difference is greatest at depth $z_{\rho}$, where

$$
\begin{aligned}
& \left|\rho_{E}\left(y, z_{\rho}\right)-\rho_{W}\left(y, z_{\rho}\right)\right| \\
& \quad=\left|\rho_{T}-\rho_{P}\right|\left(1-y / L_{y}\right) y / L_{y} \leq\left|\rho_{T}-\rho_{P}\right| / 4,
\end{aligned}
$$

the maximum being attained at $y=0.5 L_{y}$, in the middle of the basin, again in qualitative agreement with Fig. $4 c$ (quantitatively, the scaling gives a maximum eastwest density difference of a little under $1 \mathrm{~kg} \mathrm{~m}^{-3}$, in the numerical experiments, rather than the $0.6 \mathrm{~kg} \mathrm{~m}^{-3}$ of Fig. 4c). Equation (9) gives a scale for the density difference between eastern and western walls, which is significantly smaller than, but of the same order of magnitude as, the density difference between high and low latitudes; furthermore, this relationship is linear. Equation (9) thus justifies to some degree, a posteriori, the isotropy assumption underlying Bryan's (1987) use of zonal thermal wind scaling for the zonally averaged meridional flow.

The scale for the zonally integrated meridional thermal wind shear is, from Eq. (9),

$$
L_{x} \partial_{z} \bar{v} \sim-\frac{g}{f \rho_{0}}\left(\rho_{T}-\rho_{P}\right)\left(1-y / L_{y}\right) y / L_{y},
$$

where $L_{x}$ is zonal extent and the overbar marks a zonal average. Equation (10) can be integrated twice vertically to yield the meridional overturning streamfunction, as a function of latitude and depth. The necessary integration constants derive from the conditions that the vertically integrated transport is zero in the absence of wind stress, bottom topography, and momentum advection and by choosing an arbitrary value (typically zero) for the streamfunction either at the top or at the bottom. The question is what to choose for the vertical scale entering the double integral. From Eq. (8), it should be $D+\left|z_{\rho}\right|$, but this would cause problems near the equator.
Assuming an equatorial $\beta$ plane, $f=\beta y$, and noting from Eq. (6) that $z_{\rho}$ goes to zero as one approaches the equator, the zonally averaged meridional thermal wind shear and hence the overturning streamfunction would take a finite (and quite large) value at very low latitudes, instead of going to zero.

The density at the eastern boundary, as described by Eq. (7), is not compatible with vertical advective-diffusive balance, Eq. (1). The near-surface meridional density gradient implies eastward surface currents and hence downwelling in the convective region at the eastern boundary; below, flow must still be downward since stratification increases with depth. But Eq. (7) implies decreasing stratification with depth, for $z<z_{\rho}$. Hence, there must be a transition region beneath the convective zone where stratification increases with depth (implying downwelling) to then reach a maximum (implying zero vertical velocity) and decrease (implying upwelling). At high latitudes, eastward flow is stronger since the strong meridional density gradients reach deeper; hence the downwelling is stronger, and isopycnals along the eastern boundary are not flat underneath the convection zone, but slope downward with latitude (Figs. 3c and 4b). Implied is westward shear and westward flow fed by the downwelled water. Scaling suggests that the transition region has roughly the same depth as the convection zone; this assumption will be used in the following and its consequences explored. Notice that a completely self-consistent solution is not attempted here, but it is speculated that one should exist.

Assuming $2\left|z_{\rho}\right|$ for the scale of the vertical integral and integrating twice, one obtains a scale for the strength of the meridional overturning

$\Psi \sim \frac{1}{2} \frac{g}{\beta \rho_{0}} \frac{\rho_{P}-\rho_{T}}{L_{y}} 4 D^{2}\left(1-y / L_{y}\right) \ln ^{2}\left(1-y / L_{y}\right)$.

The vertical depth scale $D$ is now obtained by assuming that all net upwelling occurs uniformly in the western boundary. This means, in particular, that the $4 \mathrm{~Sv}$ that upwell at the southern boundary are neglected (see Fig. 1a). Furthermore, it is neglected that a portion of the upwelling in the western boundary is mass-balanced through the zonal overturning cell and hence does not contribute to the meridional overturning. Neither assumption is particularly well justified, but employed here for simplicity, since one now obtains from vertical advective-diffusive balance, Eq. (1),

$$
D \sim \frac{k_{v}}{w} \sim \frac{k_{v}}{\Psi_{\max }} L_{y} \Delta x,
$$

where $\Delta x$ is the zonal grid spacing. From Eq. (12) it is clear that the vertical diffusivity must be specified as a function of numerical grid size, unless the bottom or lateral boundary layer is truly resolved by the numerical grid. Therefore, $k_{v}$ must be regarded as an effective diffusivity that results from averaging over an area much larger than the one where the vigorous mixing actually 
occurs. The physical boundary layer width is almost certainly a function of the details of the mixing processes, and its discussion is beyond the scope of this paper. But it is likely that the effective mixing coefficient is a function of the large-scale solution or, in other words, that a more sophisticated and complex mixing parameterization is ultimately needed. Notice that $L_{y}$ rather than $L_{y} / 2$ appears in Eq. (12) since on zonal average downwelling occurs only in a narrow latitude band (in other words, the maximum of the meridional streamfunction occurs near the northern boundary, see the discussion below).

By combining Eqs. (11) and (12), one obtains for the meridional overturning as a function of the independent parameters and latitude

$$
\begin{aligned}
\Psi \sim & \left\{2 \frac{g}{\beta \rho_{0}} \frac{\rho_{P}-\rho_{T}}{L_{y}}\left(k_{v} \Delta x L_{y}\right)^{2}\right. \\
& \left.\times\left(1-y / L_{y}\right) \ln ^{2}\left(1-y / L_{y}\right)\right\}^{1 / 3} .
\end{aligned}
$$

To obtain an appreciation for the uncertainties involved in the expression (13), it is calculated in several steps. With the surface forcing used in the numerical experiments of section 4 , the surface density varies by about $4 \mathrm{~kg} \mathrm{~m}^{-3}$, which gives, with $\beta=2 \times 10^{-11} \mathrm{~m}^{-1}$ $\mathrm{s}^{-1}$ and $L_{y}=6000 \mathrm{~km}$,

$$
2 \frac{g}{\beta \rho_{0}} \frac{\rho_{P}-\rho_{T}}{L_{y}} \approx 6.7 \times 10^{2} \mathrm{~m} \mathrm{~s}^{-1}
$$

With $k_{v}=5 \times 10^{-4} \mathrm{~m}^{2} \mathrm{~s}^{-1}$ and $\Delta x=400 \mathrm{~km}$,

$$
\left(k_{v} \Delta x L_{y}\right)^{2} \approx 1.4 \times 10^{18} \mathrm{~m}^{8} \mathrm{~s}^{-2} .
$$

Finally, it is readily shown that

$$
\left(1-y / L_{y}\right) \ln ^{2}\left(1-y / L_{y}\right) \leq 0.54 \text {. }
$$

Multiplying (14) through (16) yields

$$
\Psi_{\max } \approx\left(520 \times 10^{18}\right)^{1 / 3} \mathrm{~m}^{3} \mathrm{~s}^{-1} \approx 8 \mathrm{~Sv},
$$

which is the correct order of magnitude but low compared to the numerical result by a factor of 2 . The typical upwelling velocity is, from the scaling of the vertical advective-diffusive balance (12), about $3.3 \times 10^{-6} \mathrm{~m}$ $\mathrm{s}^{-1}$, consistent with numerical upper-thermocline upwelling near the western boundary (Fig. 3b). The scale height of western boundary stratification, however, comes out low, as about $150 \mathrm{~m}$, perhaps because in the numerical model it is linked to the deeper easternboundary thermocline through zonal currents.

The latitudinal dependence of overturning according to the scaling (13) is plotted in Fig. 11b, together with the latitudinal dependence of overturning from the numerical experiment (Fig. 1a). Both curves are normalized so that the maximum value is 1 . Over most of the domain, the curves track each other to within 0.15 of the maximum value; the maxima coincide to within the meridional grid distance in the numerical model. This
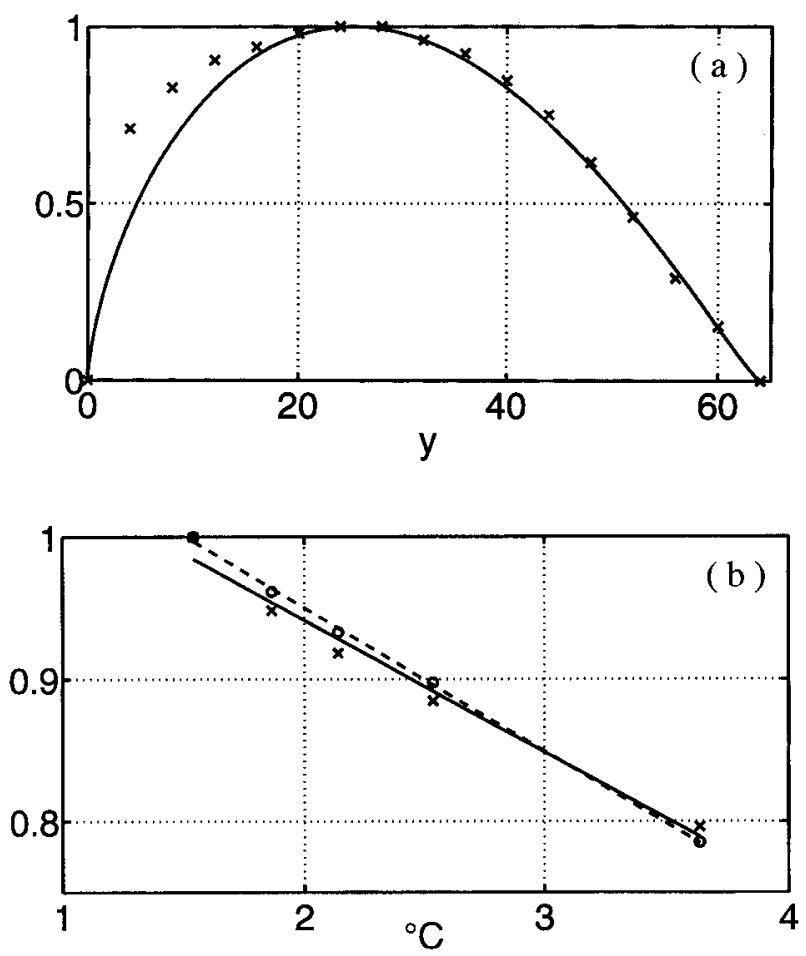

FIG. 12. (a) Latitudinal dependence of northward heat transport according to the scaling [ Eq. (13), solid] and from the numerical experiment (see Fig. 1a; boundary mixing case, $k_{v}=5 \times 10^{-4} \mathrm{~m}^{2}$ $\mathrm{s}^{-1}$; crosses). (b) Maximum heat transport from the numerical runs divided by meridional overturning at $32^{\circ} \mathrm{N}$, plotted against horizontally averaged bottom temperature from the model. Overturning at $32^{\circ} \mathrm{N}$ is taken either from the scaling [Eq. (17); crosses, solid line] or the model solution (circles, dashed line). Both sets of heat transport to overturning ratios have been normalized by their respective maximum values. The lines are least squares fits.

agreement is probably fortuitous since upwelling in the numerical model is not uniform, the vertical scale used in the thermal wind computation is not very well justified, and the meridional forcing structure in the numerical model is not linear. Nevertheless, Fig. 11b indicates that the increase of convection depth with latitude, near the eastern boundary, is indeed responsible for the fundamental dynamical balance of the meridional overturning.

When a scaling for northward heat transport is attempted according to (overturning strength) $\times$ (top-tobottom temperature contrast), good agreement with the numerical runs is again achieved in the latitudinal dependence (Fig. 12a), but this scaling cannot explain the somewhat weaker ( $1 / 2$ power) dependence on $k_{v}$, compared to the overturning. Probably, this is because surface temperature is not completely prescribed but restored to a target profile (albeit on a very short timescale). As a consequence, horizontally averaged bottom temperature is higher by over $2^{\circ} \mathrm{C}$ for the runs with large $k_{v}$ compared to the experiments with small $k_{v}$, which is consistent with the following picture.

The meridional sea surface temperature (SST) con- 
trast would be identical to the one in the restoring profile if there were no ocean currents. Meridional overturning reduces the meridional SST contrast to (Marotzke and Stone 1995)

$$
\Delta T=\left(T_{E}-T_{P}\right)\left(1-\frac{\Psi_{\max }}{V \lambda}\right),
$$

where $T_{E}$ and $T_{P}$ are respectively the equatorial and polar target temperatures from the restoring boundary condition, $V$ is high-latitude ocean volume, and $\lambda$ the inverse restoring timescale at high latitudes. Notice that although surface temperature is restored on a 30-day timescale, the effective restoring timescale of the high-latitude ocean is 100 times larger since convection makes the mixed layer reach all the way to the bottom (see Weaver et al. 1994). Hence, the ratio of high-latitude flushing timescale to effective restoring timescale is approximately

$$
\begin{aligned}
\frac{\Psi_{\max }}{V \lambda} \sim & \frac{20 \times 10^{6} \mathrm{~m}^{3} \mathrm{~s}^{-1}}{2 \times 10^{6} \mathrm{~m} \cdot 3 \times 10^{6} \mathrm{~m} \cdot 5 \times 10^{3} \mathrm{~m}} \\
& \times 3 \times 10^{8} \mathrm{~s} \sim 0.2,
\end{aligned}
$$

which is small but not completely negligible. Notice that low-latitude temperatures are reduced by the ocean heat transport, while high latitudes are warmed. In middle latitudes, where the meridional heat transport is maximum (hence its divergence zero), one can expect the midbasin SST to be identical to the target temperature. The midbasin top-to-bottom temperature contrast $\Delta T_{M}$, which enters the scaling for maximum northward heat transport, is thus approximately

$$
\Delta T_{M} \sim \frac{1}{2} \Delta T \sim \frac{1}{2}\left(T_{E}-T_{P}\right)\left(1-\frac{\Psi_{\max }}{V \lambda}\right) .
$$

Insertion of the numerical values shows that ocean heat transport reduces the vertical temperature contrast by about $1.5^{\circ} \mathrm{C}$. From the scalings (17) for $\Psi_{\max }$ and (20) for $\Delta T_{M}$, one obtains for maximum heat transport $Q_{T}$

$$
Q_{T} \propto \Psi_{M} \Delta T_{M} \propto k_{v}^{2 / 3}\left(1-\text { const } \times k_{v}^{2 / 3}\right),
$$

where $\Psi_{M}$ is midbasin meridional overturning strength. The scaling (21) exhibits weaker dependence on $k_{v}$ than to the $2 / 3$ power, consistent with the numerical results, but the good straight-line fit of squared heat transport against $k_{v}$ (Fig. 2b) might reflect that the range of steadystate solutions was insufficient to discriminate between different scaling laws. Plotting the quantity $Q_{T} k_{v}^{-2 / 3}$ as diagnosed from the numerical runs should, according to Eq. (21), yield a linear dependence on $k_{v}^{2 / 3}$, but it is more closely proportional to $k_{v}^{1 / 3}$. However, plotting $Q_{T} / \Psi_{M}$ against deep horizontally averaged temperatures from the numerical solution and $\Psi_{M}$ taken either from the scaling (17) or the model solution, gives a rather good linear dependence (Fig. 12b), thus at least confirming the first proportionality of the scaling (21).

\section{Discussion}

Mixing is employed in ocean models not only for physical reasons, but also to suppress numerical noise that would otherwise ensue, for example, when centered-difference numerics are used as in the GFDL model. In particular, Weaver and Sarachik (1990) found a spurious meridional overturning cell in the vicinity of the equator, in a model similar in geometry to the one used here; the overturning cell vanished if either vertical viscosity or the vertical resolution was increased. They also showed that if the grid Péclet number (measuring advective over diffusive fluxes on the grid scale) exceeds 2 , a computational mode can exist (similarly for viscosity, measured by the grid Reynolds number). The boundary mixing experiments presented here apparently violate this grid Péclet number criterion. However, Weaver and Sarachik (1990) stated (first sentence of their appendix) that a grid Péclet number of less that 2 is sufficient to suppress a computational mode. Conversely, a grid Péclet number of greater that 2 is a necessary (but not sufficient) condition for the existence of a computational mode. The numerical results obtained here indeed provide a counterexample: Results uncorrupted by numerical instabilities are obtained with a zero value for interior vertical mixing. This outcome, different from Weaver and Sarachik (1990), is probably the consequence of several factors.

First, following Weaver and Sarachik's (1990) arguments, a relatively high value for the vertical viscosity is employed in all experiments shown here to damp gridscale noise. Second, while the computational mode might exist, no energy is fed into it, probably because in the interior, vertical motion is entirely induced by vertical mixing (see Figs. 3a and 7c for boundary and uniform mixing, respectively). Since the geometry all by itself concentrates vertical motion near the boundaries (see again Fig. 7c), it seems more important to adhere to the grid Péclet number criterion near the boundary than in the interior. It is not clear, however, whether the experience from this idealized-geometry, buoyancy-driven model can be extrapolated to the more general case. A model with bottom topography and wind forcing could exhibit large wind-induced (external mode) vertical velocities above topographic slopes, which might feed into the computational mode if vertical diffusivity is too low. While the current results cannot be generalized, the feasibility of using physically realistic, low interior values of vertical diffusivity should be explored, rather than accepting currently used values as inevitably, numerically demanded.

A third argument is that the vertical grid used here has more evenly distributed grid spacings than Weaver and Sarachik's (1990) and, hence, is less susceptible to truncation error. Weaver et al. (1993), in their comparison of the Weaver and Sarachik (1991) and Marotzke and Willebrand (1991) experiments, concluded that the 15-level grid of the latter experiments could be used 
instead of the 33-level grid advocated by Weaver and Sarachik (1990).

Both anonymous reviewers have expressed the concern that the numerical solutions presented here, particularly the overturning strength, might be seriously compromised by truncation error arising from the vertically nonuniform grid (Yin and Fung 1991). For a variety of reasons, I do not believe that this is a serious issue. Treguier et al. (1996) have recently shown that the two main arguments of Yin and Fung (1991) against nonuniform grids are based on incorrect reasoning. First, a centered-difference scheme is second-order accurate even on a nonuniform grid; Yin and Fung (1991) used an ill-defined mapping from a regular to an irregular grid in their analysis, in which the ratio of largest to smallest vertical grid spacing diverged as the number of grid points went to infinity. Second, Yin and Fung's (1991) interpretation of second-order truncation error terms as diffusion is not justified, since these terms can be shown not to reduce temperature or salinity variance, as every diffusive term would. It is true that too irregular vertical grid spacing would lead to large truncation error (see Marti et al. 1992), but as described in the preceding paragraph, the discretization used here has proven to be reasonably smooth. In addition, while Weaver and Sarachik's (1990) solutions were strongly influenced by vertical resolution at low latitudes, their maximum overturning value (attained at high latitudes) was fairly robust $(13,15$, and $14 \mathrm{~Sv}$ for 12,19 , and 33 levels, respectively). As a last argument, notice that the irregular spacing leads to truncation error where vertical velocities are large, that is, in the vicinity of the boundaries. But there the boundary mixing model has diffusivities five times larger than the uniform-mixing version with the same averaged mixing coefficient. Hence, if any model can be expected to be robust to truncation error arising from nonuniform vertical grids, it is the boundary mixing one.

Good agreement is achieved between the numerical experiment and a theoretical analysis, which assumes (i) prescribed surface density as a function of latitude only; (ii) stable stratification in the western boundary region, following an exponential with scale D; (iii) vertical advective-diffusive balance in the grid cells at the lateral boundary, plus convection where present; and (iv) that along the eastern boundary, convection occurs down to a latitude-dependent depth $\left|z_{\rho}\right|$ and that equatorward the isopycnal is level; zonal isopycnal slopes are also assumed to vanish except in the western boundary current. The crucial departure from all previous scaling attempts is that vertical advective-diffusive balance is assumed near the boundary only and that convection is an integral part of the physical picture.

Under these assumptions, the vertical scale of density in the western boundary is related to the upwelling strength; since at the eastern boundary convection (and hence surface density) reach down to depth $\left|z_{\rho}\right|$, the eastern boundary is less dense than the western boundary.
Convective depth $\left|z_{\rho}\right|$ can be calculated as a function of latitude by following the isopycnal (assumed level) to the equator where it is level due to lack of balancing wind stress and to the outcropping latitude in the western boundary. Then, $z_{\rho}$ varies like the natural logarithm of the distance from the northern (poleward) boundary, meaning that it quickly approaches the ocean bottom at high latitudes. The density difference between eastern and western walls can then be calculated as a function of latitude and depth; its maximum value is attained at depth $\left|z_{\rho}\right|$ and is $1 / 4$ the density difference between northern and southern boundaries. This result lends some support to the often-used isotropy assumption for horizontal thermal wind shear. Thermal wind shear is converted into a transport using $2\left|z_{\rho}\right|$ for the vertical scale, and the western boundary thermocline depth D is linked, through the vertical velocity, to total meridional overturning strength. This leads to an estimate of the meridional overturning of the order of $10 \mathrm{~Sv}$, and an estimate of its meridional structure that tracks the numerical result reasonably well, including coincidence of the maxima within the discretization error. Moreover, meridional overturning scales like boundary vertical diffusivity $k_{v}$ to the $2 / 3$ power, which is remarkably well borne out by the numerical experiments. The dependence of meridional heat transport on latitude shows reasonable agreement between the scaling and the numerical runs. The experiments give a good $1 / 2$ power dependence of maximum heat transport on $k_{v}$; the scaling correctly predicts a weaker dependence on $k_{v}$ compared to the maximum overturning but does not capture the details of the dependence.

The comparison between the boundary mixing and the uniform mixing cases shows that even in the latter case, vertical motion predominantly occurs at the boundaries, induced by the density-driven mainly zonal flows. This result suggests that the boundary mixing case might be significantly easier to analyze, and less complex in its scaling behavior, than the uniform mixing model. When horizontally averaged diffusivities are identical, the uniform mixing case has an overturning strength weaker by $18 \%$ and maximum heat transport weaker by $25 \%$.

For boundary diffusivities of $30 \times 10^{-4} \mathrm{~m}^{2} \mathrm{~s}^{-1}$ and higher (no interior vertical mixing), no steady-state solutions can be found; instead, self-sustained oscillations on a roughly $25-\mathrm{yr}$ timescale ensue. It is argued that these oscillations, and possibly those in other models as well, fundamentally arise when convection reaches to the bottom all along a latitude circle, making it impossible for the vertical motion to create denser water at the western wall than at the eastern wall.

The problem of determining the density difference between eastern and western sidewalls figured prominently in the closure assumptions entering two-dimensional models of the thermohaline circulation. The problem is easier in that the complete latitude-depth structure of zonally averaged density is calculated explicitly 
by the model, so more than just the surface density is given. Still, all attempts had to resort to ill-justified or empirical arguments at some point. For example, Marotzke et al. (1988) effectively used nonrotating dynamics with artificially large viscosity (which was done from the outset by Cessi and Young 1992; Thual and McWilliams 1992; and Quon and Ghil 1992). Wright and Stocker (1991) fitted their parameterization to GCM results, as did Wright et al. (1995). The latter parameterization was considerably more sophisticated, but it made the unrealistic assumption of constant thermocline depth at the eastern boundary; moreover, neither approach included an explicit sensitivity on vertical diffusivity. Warren (1994) explicitly relied on the Stommel-Arons picture with constant diffusion; Sakai and Peltier (1995) imposed a meridional solution structure, and additionally did not obtain a dependence on vertical diffusivity. It is argued here that the assumption of zero interior vertical mixing simplifies the considerations leading to a two-dimensional closure, which hence form the analytical framework to understand the numerical model results.

I do not claim that the model used here provides a rigorous account of diapycnal mixing, even in an idealized ocean model, most notably because the horizontal diffusion implies diapycnal mixing even in the ocean interior. However, the effect does appear to be small, in the absence of a wind-driven western boundary current and associated strong isopycnal tilt. For example, with strictly zero vertical mixing everywhere, overturning strength is less than $2 \mathrm{~Sv}$, so residual diapycnal mixing is very weak. Preliminary experiments had been run with wind forcing included; then the overturning strength for zero vertical mixing was about $8 \mathrm{~Sv}$, a clear indication of horizontal diapycnal mixing. It would be interesting to repeat the experiments shown here with strictly zero diapycnal mixing in the interior, but I deemed it unnecessary for these very exploratory runs. The purpose of this paper is to suggest that, at least under some circumstances, ocean models can be run with zero interior vertical mixing, to suggest a conceptual picture of deep buoyancy-driven flows radically different from the Stommel-Arons one, and to present a theoretical analysis of the strength of the meridional overturning that is roughly consistent with the numerical experiment, both in strength and in structure. The inclusion of improved isopycnal and diapycnal mixing parameterizations, of wind forcing, and of interhemispheric flow will be left for future studies.

Acknowledgments. I wish to thank Carl Wunsch for asking me why modelers keep ignoring the observational evidence of enhanced boundary mixing and for his sustained encouragement throughout this study. The two anonymous referees provided thoughtful, constructive, and thorough reviews. Financial support was granted by the Tokyo Electric Power Company through the TEPCO/MIT Environmental Research Program, and to
$20 \%(\$ 3,000)$ jointly by the Northeast Regional Center of the National Institute for Global Environmental Change and by the Program for Computer Hardware, Applied Mathematics, and Model Physics (both with funding from the U.S. Department of Energy).

\section{REFERENCES}

Böning, C. W., W. R. Holland, F. O. Bryan, G. Danabasoglu, and J. C. McWilliams, 1995: An overlooked problem in model simulations of the thermohaline circulation and heat transport in the Atlantic Ocean. J. Climate, 8, 515-523.

Bryan, F., 1987: Parameter sensitivity of primitive equation ocean general circulation models. J. Phys. Oceanogr., 17, 970-985.

Bryan, K., 1969: A numerical method for the study of the circulation of the world ocean. J. Comput. Phys., 4, 347-376.

Cessi, P., and W. R. Young, 1992: Multiple equilibria in two-dimensional thermohaline circulation. J. Fluid Mech., 241, 291-309.

Colin de Verdière, A., 1988: Buoyancy driven planetary flows. $J$. Mar. Res., 46, 215-265.

- 1993: On the oceanic thermohaline circulation. Modelling Oceanic Climate Interactions, J. Willebrand and D. L. T. Anderson, Eds., NATO ASI Series, Springer, 151-183.

Cox, M. D., 1984: A primitive equation, three-dimensional model of the ocean. GFDL Ocean Group Tech. Rep. No. 1, GFDL/Princeton University, $40 \mathrm{pp}$. [Available from GFDL, Princeton University, P.O. Box 308, Princeton, NJ 08542.]

Cummins, P. F., G. Holloway, and A. E. Gargett, 1990: Sensitivity of the GFDL ocean general circulation model to a parameterization of vertical diffusion. J. Phys. Oceanogr., 20, 817-830.

Fanning, A. F., and A. J. Weaver, 1997: A horizontal resolution and parameter sensitivity study of heat transport in an idealized coupled climate model. J. Climate, in press.

Gargett, A. E., and G. Holloway, 1992: Sensitivity of the GFDL ocean model to different diffusivities for heat and salt. J. Phys. Oceanogr., 22, 1158-1177.

Gregg, M. C., 1987: Diapycnal mixing in the thermocline: A review. J. Geophys. Res., 92, 5249-5286.

Huang, R. X., and R. L. Chou, 1994: Parameter sensitivity of the saline circulation. Climate Dyn., 9, 391-409.

Ledwell, J. R., and B. M. Hickey, 1995: Evidence for enhanced boundary mixing in the Santa Monica Basin. J. Geophys. Res., 100, $20665-20679$.

- and A. Bratkovich, 1995: A tracer study of mixing in the Santa Cruz Basin. J. Geophys. Res., 100, 20 681-20 704.

_ A. J. Watson, and C. S. Law, 1993: Evidence for slow mixing across the pycnocline from an open-ocean tracer-release experiment. Nature, 364, 701-703.

Marotzke, J., 1990: Instabilities and multiple equilibria of the thermohaline circulation. Ph.D. thesis. Berichte Institut für Meereskunde Kiel, 194, 126 pp. [Available from J. Marotzke, MIT, Room 54-1514, Cambridge, MA 02139.]

_ 1991: Influence of convective adjustment on the stability of the thermohaline circulation. J. Phys. Oceanogr., 21, 903-907. , and J. Willebrand, 1991: Multiple equilibria of the global thermohaline circulation. J. Phys. Oceanogr., 21, 1372-1385.

— circulation, and flux adjustments in a simple coupled model. $J$. Phys. Oceanogr., 25, 1350-1364.

_ , P. Welander, and J. Willebrand, 1988: Instability and multiple steady states in a meridional-plane model of the thermohaline circulation. Tellus, 40A, 162-172.

Marti, O., G. Madec, and P. Delecluse, 1992: Comment on "Net diffusivity in ocean general circulation models with nonuniform grids" by F. L. Yin and I. Y. Fung. J. Geophys. Res., 97, 12 76312766.

Munk, W., 1966: Abyssal recipes. Deep-Sea Res., 13, 707-730.

Quon, C., and M. Ghil, 1992: Multiple equilibria in thermosolutal 
convection due to salt-flux boundary conditions. J. Fluid Mech., 245, 449-483.

Sakai, K., and W. R. Peltier, 1995: A simple model of the Atlantic thermohaline circulation: Internal and forced variability with paleoclimatic implications. J. Geophys. Res., 100, 13 455-13 479.

Samelson, R. M., 1997: Large-scale circulation with locally enhanced vertical mixing. J. Phys. Oceanogr.

Schiff, L. I., 1966: Lateral boundary mixing in a simple model of ocean convection. Deep-Sea Res., 13, 621-626.

Stommel, H., 1958: The abyssal circulation. Deep-Sea Res., 5, 8082.

- and A. B. Arons, 1960a: On the abyssal circulation of the world ocean-I. Stationary planetary flow patterns on a sphere. DeepSea Res., 6, 140-154.

- and 1 1960b: On the abyssal circulation of the world ocean-II. An idealized model of the circulation pattern and amplitude in oceanic basins. Deep-Sea Res., 6, 217-233.

,-- , and A. J. Faller, 1958: Some examples of stationary planetary flow patterns in bounded basins. Tellus, 10, 179-187.

Thual, O., and J. C. McWilliams, 1992: The catastrophe structure of thermohaline convection in a two-dimensional fluid model and a comparison with low-order box models. Geophys. Astrophys. Fluid Dyn., 64, 67-96.

Treguier, A. M., J. K. Dukowicz, and K. Bryan, 1996: Properties of nonuniform grids used in ocean general circulation models. $J$. Geophys. Res., 101, 20 877-20 881.

Warren, B. A., 1994: Driving the meridional overturning in the Indian Ocean. Deep-Sea Res., 41, 1349-1360.

Weaver, A. J., and E. S. Sarachik, 1990: On the importance of vertical mixing in certain ocean general circulation models. J. Phys. Oceanogr., 20, 600-609.
- , and - 1991: The role of mixed boundary conditions in numerical models of the ocean's climate. J. Phys. Oceanogr., 2l, 1470-1493.

_ - J. Marotzke, P. F. Cummins, and E. S. Sarachik, 1993: Stability and variability of the thermohaline circulation. J. Phys. Oceanogr., 23, 39-60.

— S. M. Aura, and P. G. Myers, 1994: Interdecadal variability in an idealized model of the North Atlantic. J. Geophys. Res., 99, 12 423-12 441.

Welander, P., 1971: The thermocline problem. Philos. Trans. Roy. Soc. London, A270, 415-421.

Winton, M., 1996: The role of horizontal boundaries in parameter sensitivity and decadal-scale variability of coarse-resolution ocean general circulation models. J. Phys. Oceanogr., 26, 289-304.

Wright, D. G., and T. F. Stocker, 1991: A zonally averaged ocean model for the thermohaline circulation. Part I: Model development and flow dynamics. J. Phys. Oceanogr., 21, 1713-1724.

- C. B. Vreugdenhil, and T. M. C. Hughes, 1995: Vorticity dynamics and zonally averaged ocean circulation models. J. Phys. Oceanogr., 25, 2141-2154.

Wunsch, C., 1970: On oceanic boundary mixing. Deep-Sea Res., 17, 293-301.

Yin, F. L., and I. Y. Fung, 1991: Net diffusivity in ocean general circulation models with nonuniform grids. J. Geophys. Res., 96, $10773-10776$.

Zhang, J., R. W. Schmitt, and R. X. Huang, 1997: Sensitivity of the GFDL modular ocean model to the parameterization of double-diffusive processes. J. Phys. Oceanogr.

Zhang, S., C. A. Lin, and R. J. Greatbatch, 1992: A thermocline model for ocean-climate studies. J. Mar. Res., 50, 99-124. 